\title{
Stationary Random Fields Arising From Second-Order Partial Differential Equations on Compact Lie Groups
}

\author{
Salem Said, Pierre-Olivier Amblard, and Jonathan H. Manton, Senior Member, IEEE
}

\begin{abstract}
Wide sense stationary processes are a mainstay of classical signal processing. It is well known that they can be obtained by solving ordinary differential equations with constant coefficients whose right-hand side is a white noise. This paper addresses the extension of this construction to random fields defined on compact Lie groups. On an underlying compact Lie group, the paper studies left invariant second-order elliptic partial differential equations whose right-hand side is a spatial white noise. Quite often, the solution of a partial differential equation is not defined as a function but as a distribution. To adapt to this situation, the paper introduces a definition of wide sense stationary distributions on a compact Lie group. This is shown to be consistent with the more restricted definition of wide sense stationary fields given in a classic paper by Yaglom. It is proved that the solution of a partial differential equation, of the kind being studied, is a wide sense stationary distribution whose covariance structure is determined by the fundamental solution of the equation. As a concrete example, this paper describes the fundamental solution of the Helmholtz equation on the rotation group and the resulting covariance structure.
\end{abstract}

Index Terms - Lie group, partial differential equation, random field, white noise, wide sense stationary.

\section{INTRODUCTION}

$\mathbf{T}$ HE main themes of this paper are partial differential equations and wide sense stationarity. For shortness, "partial differential equation" (or "equations") will be abbreviated to PDE and "wide sense stationary" to WSS.

WSS processes are among the most popular objects in signal processing and data analysis [1], [2]. There are many reasons for this. Of practical importance among them is that so-called finite-order processes belong to the class of WSS processes.

A WSS process $\xi$ consists of complex ${ }^{1}$ random variables $\xi(t)$ for $t \in \mathbb{R}$, where $t$ is understood as time. Stationarity refers to

Manuscript received July 23, 2012; revised December 11, 2012; accepted January 15,2013 . Date of publication January 28, 2013; date of current version May 15, 2013. P.-O. Amblard was supported by a Marie Curie International Outgoing Fellowship from the European Community.

S. Said and J. H. Manton are with the Department of Electrical and Electronic Engineering, The University of Melbourne, Parkville, Vic. 3010, Australia (e-mail: ssaid@unimelb.edu.au; j.manton@ieee.org).

P.-O. Amblard is with the Department of Electrical and Electronic Engineering, The University of Melbourne, Parkville, Vic. 3010, Australia, and also with the GIPSA-lab UMR 5216, Centre National de la Recherche Scientifique, 38402 Saint Martin d'Hères, France (e-mail: bamblard@unimelb.edu.au).

Communicated by G. V. Moustakides, Associate Editor for Detection and Estimation.

Digital Object Identifier 10.1109/TIT.2013.2243202

${ }^{1}$ In this paper, only scalar (univariate) random objects are considered. the fact that the covariance structure of $\xi$ is time homogeneous. That is, for $t, s \in \mathbb{R}$

$$
\mathbb{E} \xi(t) \xi^{*}(s)=B(t-s)
$$

where * denotes complex conjugation. Here, $B$ is called the covariance function of $\xi$ and is, in fact, the main object used to understand the process $\xi$; it is either assumed $\mathbb{E} \xi(t) \equiv 0$ identically, or that $\mathbb{E} \xi(t)$ is constant and of no importance. In general, WSS processes give rise to $B$ which is continuous and positive definite. Bochner's theorem states that any such function is the Fourier transform of a finite measure. In signal processing, this measure is known as the spectral measure or spectral power distribution of $\xi$.

Finite-order processes are straightforward extensions of the well-known stationary Ornstein-Uhlenbeck process. This process is realized by letting $\xi$ be the steady-state solution of an ordinary differential equation

$$
\frac{d}{d t} \xi+\beta \xi=w
$$

where $\beta>0$ and $w$ is a so-called white noise process. In other words, $\xi$ is obtained as the output of a first-order filter whose input is $w$. Formally, $w$ is a real WSS process whose covariance function is given by

$$
\mathbb{E} w(t) w(s)=\delta(t-s)
$$

where $\delta$ is the Dirac delta "function." Now, this is only a formal definition. The "function" $\delta$ is not a function and a fortiori not a continuous positive-definite function.

Finite-order processes are WSS processes which arise as solutions of ordinary differential equations with constant coefficients whose right-hand side is a white noise process. The above equation clearly falls within this definition. In order to consider such equations systematically, a clear meaning needs to be given to the concept of white noise process. A complete approach can be developed based on some elementary aspects of the theory of distributions, (the reader may wish to recall [3] for a general introduction to distributions).

First, the Bochner-Minlos theorem shows that a white noise process can be understood as a random tempered distribution [4]. Tempered distributions allow for the full application of Fourier transform methods. For any such distribution in the 
right-hand side of an ordinary differential equation with constant coefficients, Fourier analysis can be used to represent the solution both in spectral form (i.e., Fourier representation) and as a convolution with the fundamental solution. Thus, each realization of a white noise is a tempered distribution to which these two representations apply. The spectral representation is particularly helpful as it gives the spectral power distribution of the solution.

This paper aims to extend the situation described for finite-order processes to random fields defined on compact Lie groups. WSS random fields on Lie groups, or even topological groups in general, were studied in [5]. They were shown to appear in a variety of applications and to admit useful generalizations of common signal-processing techniques. In [6], estimation and detection problems for fields on compact Lie groups were solved and applied to vision and object detection problems. In spite of WSS random fields on Lie groups appearing in these and other applications, it seems there are not many simple mathematical models or constructions of these WSS random fields available in the literature. The motivation of this paper has been to overcome this problem by showing that certain WSS fields on compact Lie groups can be constructed as solutions to invariant PDE.

In his 1961 paper [7], Yaglom considered the generalization of WSS processes to WSS random fields defined on groups. This generalization has both applied and theoretical significance. A group $G$ has a, generally noncommutative but always associative, operation which gives for elements $g, h \in G$ their "composition" $g h \in G$. It also possesses and identity element $1 \in G$ such that $g 1=1 g=g$ for $g \in G$ and an inverse element $g^{-1}$ for each $g \in G$ which satisfies $g^{-1} g=g g^{-1}=1$. Of course, $\mathbb{R}$ itself is a group where the composition of $t, s \in \mathbb{R}$ is $t+s$ and the identity element is the number 0 .

Instead of random variables $\xi(t)$ for $t \in \mathbb{R}$, Yaglom considered random variables $\xi(g)$ for $g \in G$. These form a random field $\xi$ and it is natural to take $G$ to be a topological group (usually, any group structure of interest corresponds to some standard topology). Now, due to the fact that $G$ may be noncommutative, there are two ways in which $\xi$ can be WSS, (homogeneous, in Yaglom's terminology). Precisely, $\xi$ is called left WSS if, for $g, h \in G$

$$
\mathbb{E} \xi(g) \xi^{*}(h)=B\left(h^{-1} g\right)
$$

and it is called right WSS if

$$
\mathbb{E} \xi(g) \xi^{*}(h)=B\left(g h^{-1}\right)
$$

in either case, $B$ is known as the covariance function of $\xi$ and again becomes the main object of study. In a sense explained by Yaglom, $B$ is a continuous positive-definite function on $G$. If $G$ is a so-called type I separable topological group, then a generalization of Bochner's theorem gives rise to an operator-valued measure which plays the role of spectral power distribution. This is the main fact used by Yaglom and it is also central in the signal-processing techniques proposed in [5].

In this paper, the group $G$ is assumed to be a compact Lie group. This restriction is aimed at profiting from the powerful yet simple tools of harmonic analysis on compact Lie groups.
It seems that most of the following can be generalized to semisimple noncompact Lie groups (which fall within type I groups mentioned above), however, that would require significantly more difficult aspects of functional analysis. Harmonic analysis on compact Lie groups has been applied in many engineering papers [8]-[10]. Here, the required background is given in Appendix A, which also defines the notation used throughout this paper.

This paper will study linear PDE on the underlying group $G$ whose right-hand side is a spatial white noise. In order for the solutions of these equations to be WSS, the equations themselves should respect the group structure. As mentioned previously, it is possible to consider either left or right WSS. The difference between the two is trivial. Indeed, if $\xi$ is a left WSS field, then $\tilde{\xi}$ given by $\tilde{\xi}(g)=\xi^{*}\left(g^{-1}\right)$ is right WSS. To avoid unnecessary digressions, this paper only considers the left WSS case and the mention of "left" is dropped. The required PDE takes the form

$$
\mathcal{L} u=w
$$

where $\mathcal{L}$ is a left invariant differential operator and $w$ a spatial white noise. Formally, $w$ is a WSS random field where

$$
\mathbb{E} w(g) w^{*}(h)=\delta\left(h^{-1} g\right)
$$

again $\delta$ is the Dirac delta, but on the group $G$. To keep the paper to a reasonable length, the operator $\mathcal{L}$ is restricted to being a second-order elliptic operator. The aim is to show the behavior of the above PDE is analogous to that of ordinary differential equations leading to finite-order processes. This is carried out in several steps which generally correspond to the various sections of this paper.

Section II reviews the above PDE problem in the deterministic case. The solution is first defined as a distribution, i.e., as a weak solution. By introducing Sobolev spaces of positive and negative order, the regularity of the solution is related to that of the right-hand side; see Proposition 3. It is also shown that the solution is the convolution of the right-hand side with the fundamental solution. This reflects the fact that the PDE is left invariant.

The approach of Section II is used as a model for studying the white noise PDE. To include white noise into a similar framework, it seems the definition of WSS should be extended from fields to distributions defined on $G$. Indeed, the formal definition of spatial white noise suggests it is typically a very irregular object. Thus, one expects it to be defined only as a distribution, not a function.

This extension is carried out in Section III. This section introduced the new concept of a WSS distribution. A WSS distribution $u$ is characterized by its covariance $B$; however, $B$ is a distribution and not a function in general. In connection to the deterministic PDE case, WSS distributions are defined as elements of random Sobolev spaces. They satisfy a spectral characterization which generalizes the one given in [7] - this is stated in Theorem 3. Spatial white noise itself appears as a WSS distribution $w$ whose covariance is the Dirac delta. Its definition is recovered in Theorem 5.

With the results of Sections II and III, the full white noise PDE is finally considered in Section IV. Its solution is shown to 
be a WSS distribution with covariance determined by the fundamental solution of the deterministic equation. This is given in Proposition 6. In fact, just as for the deterministic equation considered in Section II, the solution of the white noise PDE is given by the convolution of the white noise $w$ with the fundamental solution.

Under some additional conditions, the solution is a square integrable field, as in the classical definition of Yaglom. For square integrable random fields, Theorem 4 of Section III states that weak (i.e., distributional) derivatives coincide with derivatives in the square mean. This means that the white noise PDE can be entirely understood in the square mean sense. Incidentally, this is a usual point of view for ordinary differential equations used to obtain WSS processes.

This paper closes with Sections V and VI. Section V provides a specific example of the white noise PDE studied in Section IV. Namely, it considers the Helmholtz equation on the special orthogonal group $S O(3)$ (i.e., the rotation group of $\mathbb{R}^{3}$ ). It is shown the fundamental solution of this equation is a square integrable function, which is given by the density of the Laplace distribution, known in directional statistics. Accordingly, the solution of the white noise PDE is a square integrable field. Section VI gives a final discussion of the results in previous sections. It also describes some important open problems arising from these results.

\section{Deterministic ElLiptic EQuATION}

In order to study a stochastic system, it is often helpful to have a good understanding of a similar deterministic system. The approach taken in Section IV for the white noise PDE is closely based on the following approach for a deterministic PDE.

Recall now $G$ is a compact Lie group. Let $\mathfrak{g}$ be its Lie algebra, whose elements are identified with left invariant vector fields. With respect to some Ad-invariant scalar product, let $X_{1}, \ldots, X_{d}$ be a fixed orthonormal basis of $\mathfrak{g}$, (see Appendix A for a more detailed introduction). The equation of interest takes the form

$$
\mathcal{L} u=\omega
$$

for an unknown $u$ and a given right-hand side $\omega$, where the differential operator $\mathcal{L}$ is a second-order elliptic differential operator. Precisely

$$
\mathcal{L}=a+\sum_{i=1}^{d} b_{i} X_{i}+\sum_{i, j=1}^{d} c_{i j} X_{i} X_{j}
$$

where $a<0, b_{i} \in \mathbb{R}$, and $\left(c_{i j}\right)$ is a real symmetric strictly positive-definite matrix. It will be suitable to take $\omega \in D^{\prime}(G)$ and search for the solution $u \in D^{\prime}(G)$; here, $D^{\prime}(G)$ is the space of distributions introduced in Section II-A. This separates the question of existence and uniqueness of a solution $u$ from the question of regularity properties of this solution.

In the following, Section II-A introduces a scale (a nested sequence) of Sobolev spaces suitable for characterizing the regularity of solutions of (1). Existence and uniqueness of solutions is given in Section II-B2. In particular, $u$ is shown to be given by the convolution of $\omega$ with a fundamental solution $e$. This is also a distribution, $e \in D^{\prime}(G)$; see (36). Regularity properties of $u$ are closely related to the fact that $\mathcal{L}$ is elliptic. They are given in Proposition 3.

\section{A. Sobolev Spaces and Regularity}

Sobolev spaces are here introduced to describe the regularity properties of solutions of (1). In general, Sobolev spaces are highly useful to the study of elliptic PDE, whether in Euclidean space or in other differentiable manifolds. Essentially, they are Hilbert spaces and thus facilitate the variational formulation of a PDE, replacing it by the problem of minimizing a quadratic energy integral.

The Sobolev spaces considered here are $H_{n}(G)$ where $n \in \mathbb{Z}$. For positive $n$, these are spaces of functions $f \in L^{2}(G)$, with additional regularity properties. The space $H_{-n}(G)$ is identified with the dual space of $H_{n}(G)$. Thus, for $n$ positive, $H_{-n}(G)$ is not a space of functions but of distributions. Whether $n$ is positive or negative, $H_{n}(G)$ is a Hilbert space. The following presentation is similar to that of $[11, \mathrm{Ch} .10]$. For easier reference, it is given in a relatively detailed form. A general source on functional analysis and PDE is [12]. Appendix B presents an elementary example of the following discussion and can be used for motivation.

As usual when considering PDE, it is suitable to consider a sufficiently large space of distributions. First, the space of test functions needs to be introduced. As a set, this is the space $D(G)$ of functions $k \in C^{\infty}(G)$. It is moreover equipped with the topology of the following seminorms:

$$
|k|_{n}=\sum_{|\beta| \leq n} \sup \left|X^{\beta} k\right| \quad n \in \mathbb{N}
$$

where $\beta=\left(\beta_{1}, \ldots, \beta_{d}\right) \in \mathbb{N}^{d}$ and $|\beta|=\beta_{1}+\cdots+\beta_{d}$. The supremum is taken over $g \in G$ in the expression

$$
X^{\beta} k(g)=X_{1}^{\beta_{1}} \ldots X_{d}^{\beta_{d}} k(g)
$$

which should be understood as the definition of the notation $X^{\beta}$, (i.e., the symbol $X$ by itself has no meaning here).

The space of distributions $D^{\prime}(G)$ is the dual space of $D(G)$. Thus, $u \in D^{\prime}(G)$ is a linear functional $u: D(G) \rightarrow \mathbb{C}$ such that, for some $n \in \mathbb{N}$ and $C>0$

$$
|u(k)| \leq C|k|_{n} \quad k \in D(G) .
$$

This property is equivalent to another one which involves convergence. In fact, $u$ verifies (4) iff, for $k, k_{1}, k_{2}, \cdots \in D(G)$, the complex numbers $u\left(k_{i}\right)$ converge to $u(k)$ whenever the functions $X^{\beta} k_{i}$ for $|\beta| \leq n$ converge uniformly to $X^{\beta} k$. Note here that $\beta=(0, \ldots, 0)$ for which $X^{\beta} k=k$ is included in the condition $|\beta| \leq n$.

Here are two fundamental examples to keep in mind. First, note that $f \in L^{2}(G)$ defines a distribution $u_{f}$

$$
u_{f}(k)=\langle k, f\rangle
$$

where (4) is verified with $n=0$ and $C=\|f\|$. The second example is the famous Dirac delta

$$
\delta(k)=k(1)
$$

here also, (4) is verified for $n=0$. As of now, $f$ is identified with $u_{f}$ and both are indiscriminately written $f$.

As already stated, studying (1) in $D^{\prime}(G)$ separates the problem of finding a solution from the problem of knowing the 
regularity (i.e., differentiability) of this solution. Concretely, this is done by searching for $u \in D^{\prime}(G)$ which is a weak solution of (1). This requires the concept of weak derivative.

Let $X \in \mathfrak{g}$, the weak derivative $X u \in D^{\prime}(G)$ of $u \in D^{\prime}(G)$ is again a distribution

$$
X u(k)=-u(X k) \quad k \in D(G)
$$

In the case where $u=f$ this is just (94) in Appendix A. Again, (4) is clearly satisfied. If $u$ satisfies (4) for $n$ and $C$ then $X u$ satisfies (4) for $n+1$ and $C$. Higher order derivatives $X^{\beta} u$ are defined as successive weak derivatives. Thus

$$
X^{\beta} u(k)=(-1)^{|\beta|} u\left(X^{\beta} k\right) \quad k \in D(G) .
$$

It is now possible to introduce the Sobolev spaces $H_{n}(G)$. Since $f \in L^{2}(G)$ has been identified with $u_{f} \in D^{\prime}(G)$ as in (5), it is then possible to speak of the weak derivatives of $f$. Precisely, let $X^{\beta} f^{\prime} \in D^{\prime}(G)$ be defined as in (8). For $n \in \mathbb{N}$, the space $H_{n}(G)$ is the space of all those $f \in L^{2}(G)$ such that $X^{\beta} f \in L^{2}(G)$ for $|\beta| \leq n$. In particular, this definition implies $H_{n}(G) \subset$ $L^{2}(G) \subset D^{\prime}(G)$.

To further clarify this definition, consider the example of $H_{1}(G)$. This is the space with elements $f \in L^{2}(G)$ such that there exist $X_{1} f, \ldots, X_{d} f \in L^{2}(G)$ with the property

$$
\left\langle k, X_{i} f\right\rangle=-\left\langle X_{i} k, f\right\rangle \quad k \in D(G) .
$$

It is not difficult to see that $H_{n}(G)$ for $n \in \mathbb{N}$ can be given the topology of a Hilbert space using the inner product

$$
\left(f_{1}, f_{2}\right)_{n}=\sum_{\langle\beta| \leq n}\left\langle X^{\beta} f_{1}, X^{\beta} f_{2}\right\rangle
$$

for $f_{1}, f_{2} \in H_{n}(G)$. It can be shown that an equivalent inner product, i.e., one that gives the same topology for $H_{n}(G)$, is given by (see [11] or compare to (102) in Appendix A)

$$
\left\langle f_{1}, f_{2}\right\rangle_{n}=\sum_{\pi} d_{\pi}\left(1+\kappa_{\pi}\right)^{n} \operatorname{tr}\left\{\hat{f}_{1}(\pi) \hat{f}_{2}^{\dagger}(\pi)\right\}
$$

where $\kappa_{\pi}$ are (up to sign) the eigenvalues of the Laplace operator, defined in (100). The corresponding norm will be denoted $\|f\|_{n}$.

Going on with $n \in \mathbb{N}$, recall $H_{n}(G)$ has been defined as a Hilbert space. This means that it is isomorphic to its dual space. However, this dual space can be identified with a larger subspace of $D^{\prime}(G)$, denoted $H_{-n}(G)$. This is now developed briefly.

Note that $u \in D^{\prime}(G)$ is uniquely determined by the matrices $\hat{u}(\pi)$, where for $\pi \in \hat{G}$

$$
\hat{u}^{\dagger}(\pi)=u\left(U^{\pi}\right)
$$

These matrices will be called Fourier coefficients of $u$. Their definition is quite similar to that of the Fourier transform of distributions (compare to [3, p. 189]).

For $k \in D(G)$, the uniform convergence of the Fourier series (90) for all $f=X^{\beta} k$ implies by application of the remark after

$$
u(k)=\sum_{\pi} d_{\pi} \operatorname{tr}\left\{\hat{k}(\pi) \hat{u}^{\dagger}(\pi)\right\}
$$

That is, $u$ can be applied to the Fourier series (90) of $k$ term by term.

It is possible to show, using classical arguments as in [12], that for $n \in \mathbb{Z}$ and $u \in D^{\prime}(G)$, the following condition is equivalent to $u \in H_{n}(G)$. Precisely

$$
\|u\|_{n}^{2}=\sum_{\pi} d_{\pi}\left(1+\kappa_{\pi}\right)^{n} \operatorname{tr}\left\{\hat{u}(\pi) \hat{u}^{\dagger}(\pi)\right\}<\infty .
$$

Moreover, given any matrices $\hat{u}(\pi)$ which verify this condition, these define a unique $u \in H_{n}(G)$ as in (13). Norm (14) arises from an inner product similar to $(11)$, since it is possible to allow negative $n$ in that formula.

The duality between $H_{-n}(G)$ and $H_{n}(G)$ is given by the same formula (13). Precisely, if $u$ is replaced by $u_{1} \in H_{-n}(G)$ and $k$ is replaced by $u_{2} \in H_{n}(G)$, then the resulting $u_{1}\left(u_{2}\right)$ verifies

$$
\left|u_{1}\left(u_{2}\right)\right| \leq\left\|u_{1}\right\|_{-n}\left\|u_{2}\right\|_{n}
$$

This is simply a result of (14) and the Cauchy-Schwarz inequality.

The definition of $H_{n}(G)$ by means of (14) plays an important role in the following result.

Theorem 1 (Sobolev Embedding Theorem): The following inclusions are compact with dense range:

$$
H_{n}(G) \subset L^{2}(G) \subset H_{-n}(G)
$$

for $n \in \mathbb{N}$. Moreover, the spaces $D(G)$ and $D^{\prime}(G)$ can be obtained as follows:

$$
D(G)=\bigcap_{n \in \mathbb{N}} H_{n}(G) \quad D^{\prime}(G)=\bigcup_{n \in \mathbb{N}} H_{-n}(G) .
$$

The Sobolev embedding theorem states that each $f \in H_{n}(G)$ where $n>k+d / 2$ has a modification which is in $C^{k}(G)$; here, $k \in \mathbb{N}$ and $C^{0}(G)=C(G)$ by convention.

This theorem can be used to give a characterization of the spaces $D(G)$ and $D^{\prime}(G)$ similar to the characterization (14). Indeed, it results from (14) and (17) that a linear functional $u$ : $D(G) \rightarrow \mathbb{C}$ verifies $u \in D^{\prime}(G)$ iff the coefficients (12) verify

$$
\operatorname{tr}\left\{\hat{u}(\pi) \hat{u}^{\dagger}(\pi)\right\}=o\left(\kappa_{\pi}^{n}\right) \text { for some } n \in \mathbb{N} \text {. }
$$

On the other hand, it follows from the Sobolev embedding theorem that $u \in D^{\prime}(G)$ is equivalent through (5) to $f \in D(G)$ iff

$$
\operatorname{tr}\left\{\hat{u}(\pi) \hat{u}^{\dagger}(\pi)\right\}=o\left(\kappa_{\pi}^{-n}\right) \quad \text { for all } n \in \mathbb{N} \text {. }
$$

Conversely, if some matrices $\hat{u}(\pi)$ verify (18) or (19), then these define a unique $u$ in $D^{\prime}(G)$ or $D(G)$, respectively. These two conditions will shortly be applied in defining the convolution of distributions.

\section{B. Convolution and Fundamental Solution}

It is a known fact, at least for PDE with constant coefficients in Euclidean space, that the solution of a nonhomogeneous linear elliptic equation is given by the convolution of its right-hand side with its fundamental solution. This will also be the case for the (1). Moreover, quite similar to an equation in 
Euclidean space, the fundamental solution is obtained from the Fourier representation of the equation, in other words of the operator $\mathcal{L}$.

1) Convolution of Distributions: Recall first the concept of convolution of two functions $f_{1}, f_{2} \in L^{2}(G)$. This is $f_{2} * f_{1} \in$ $L^{2}(G)$, where

$$
\left(f_{2} * f_{1}\right)(g)=\int f_{1}\left(g h^{-1}\right) f_{2}(h) d h .
$$

Now a usual application of (89) yields the convolution theorem [11], [13]

$$
\widehat{f_{2} * f_{1}}(\pi)=\hat{f}_{2}(\pi) \hat{f}_{1}(\pi) \text {. }
$$

In order to write down the solution of (1) in the form of a convolution, it is necessary to define the convolution of distributions. First, let $u \in D^{\prime}(G)$ and $k \in D(G)$. Consider the following function:

$$
(u * k)(g)=u\left(\tilde{k}_{g}\right)^{*} \text {. }
$$

Here, $\tilde{k}_{g} \in D(G)$ is given by $\tilde{k}_{q}(h)=k^{*}\left(g h^{-1}\right)$. It can be shown using (4) that $u * k \in C(G)$. To show that $u * k \in D(G)$, apply (12) and (13) to obtain

$$
\widehat{u * k}(\pi)=\hat{u}(\pi) \hat{k}(\pi) .
$$

Since $u$ verifies (18) and $k$ verifies (19), it follows $u * k$ verifies (19), so $u * k \in D(G)$.

Now, if $u_{1}, u_{2} \in D^{\prime}(G)$, there is a unique $u_{2} * u_{1} \in D^{\prime}(G)$ such that for all $k \in D(G)$

$$
\left(u_{2} * u_{1}\right) * k=u_{2} *\left(u_{1} * k\right) .
$$

It follows from (12), (23), and (24) that $u_{1} * u_{2}$ is characterized by

$$
\widehat{u_{2} * u_{1}}(\pi)=\hat{u}_{2}(\pi) \hat{u}_{1}(\pi) .
$$

The following proposition gives a regularizing property of convolution.

Proposition 1 (Regularizing Property): If $u_{1} \in H_{n}(G)$ and $u_{2} \in H_{m}(G)$, where $n, m \in \mathbb{Z}$, then $u_{2} * u_{1} \in H_{n+m}(G)$.

This proposition will not play a major role in the following. However, it is an interesting application of (14) and (25). In order to prove $u=u_{1} * u_{2} \in H_{n+m}(G)$, it is enough to prove $u$ verifies condition (14). By (25)

$$
\operatorname{tr}\left\{\hat{u}(\pi) \hat{u}^{\dagger}(\pi)\right\} \leq \operatorname{tr}\left\{\hat{u}_{2}(\pi) \hat{u}_{2}^{\dagger}(\pi)\right\} \operatorname{tr}\left\{\hat{u}_{1}(\pi) \hat{u}_{1}^{\dagger}(\pi)\right\} .
$$

By replacing in the sum which appears in (14)

$$
\|u\|_{n+m}^{2} \leq\left\|u_{2}\right\|_{m} \sup _{\pi}\left(1+\kappa_{\pi}\right)^{n} \operatorname{tr}\left\{\hat{u}_{1}(\pi) \hat{u}_{1}^{\dagger}(\pi)\right\}
$$

since $u_{1} \in H_{n}(G)$, the supremum over $\pi \in \hat{G}$ is finite. Since $u_{2} \in H_{m}(G)$, it follows $\|u\|_{n+m}<\infty$. Thus, $u$ verifies condition (14) so that $u_{2} * u_{1} \in H_{n+m}(G)$.

2) Fundamental Solution: It is now possible to tackle the (1). As already stated, the approach will be to search for $u$ which is a weak solution. Once this is found, its regularity is determined by finding $n$ such that $u \in H_{n}(G)$ and applying the Sobolev embedding theorem. A distribution $u \in D^{\prime}(G)$ is called a weak solution of (1) if the equation holds in $D^{\prime}(G)$ with $\mathcal{L} u$ interpreted as in (8).

By taking Fourier coefficients of both sides of (1), the equation is reduced to a family of linear matrix equations. The central role is played by the symbol of the operator $\mathcal{L}$. This is the family of matrices $\hat{\mathcal{L}}_{\pi}$ where

$$
\mathcal{L}\left(U^{\pi}\right)=U^{\pi} \hat{\mathcal{L}}_{\pi} \pi \in \hat{G} .
$$

The properties of the symbol of $\mathcal{L}$ are given in the following proposition.

Proposition 2: Let $\hat{\mathcal{L}}_{\pi}$ be given by (26). Then, $\hat{\mathcal{L}}_{\pi}$ is invertible for all $\pi \in \hat{G}$. Moreover, let $b(\pi)$ be the inverse of $\hat{\mathcal{L}}_{\pi}^{\dagger} \hat{\mathcal{L}}_{\pi}$. Whenever $\pi \neq \pi_{0}$, the spectral radius $\varepsilon_{\pi}$ of $b(\pi)$ verifies $\varepsilon_{\pi} \leq \alpha \kappa_{\pi}^{-2}$, where $\alpha>0$ does not depend on $\pi$.

Proof: First, note that $\hat{\mathcal{L}}_{\pi_{0}}=a$. Since $a<0$, this is invertible. Consider the case $\pi \neq \pi_{0}$. Using (96) and (97)

$$
\hat{\mathcal{L}}_{\pi}=\sum_{i=1}^{d} b_{i} X_{i}^{\pi}+a I_{\pi}+\sum_{i, j=1}^{d} c_{i j} X_{i}^{\pi} X_{j}^{\pi} .
$$

Let $D_{\pi}$ be given by

$$
D_{\pi}=\sum_{i, j=1}^{d} c_{i j} X_{i}^{\pi} X_{j}^{\pi}
$$

Since $\left(c_{i j}\right)$ is symmetric strictly positive definite, there exists an orthonormal basis $Z_{1}, \ldots, Z_{d}$ of $\mathfrak{g}$ such that

$$
D_{\pi}=\sum_{i=1}^{d} \gamma_{i} Z_{i}^{\pi} Z_{i}^{\pi}
$$

where $0<\gamma_{1} \leq \cdots \leq \gamma_{d}$ are the eigenvalues of $\left(c_{i j}\right)$. Since the $X_{i}^{\pi}$ and $Z_{i}^{\pi}$ are skew Hermitian, it follows the first term in (27) is skew Hermitian while the second and the third are Hermitian strictly negative definite. In particular, for $z \in \mathbb{C}^{d_{\pi}}$

$$
\Re\left(z^{\dagger} \hat{\mathcal{L}}_{\pi} z\right)=z^{\dagger}\left(a I_{\pi}+D_{\pi}\right) z<0
$$

where $\Re$ denotes the real part. Thus, $\hat{\mathcal{L}}_{\pi}$ is invertible.

Let $\rho_{\pi}>0$ be the smallest eigenvalue of $\hat{\mathcal{L}}_{\pi}^{\dagger} \hat{\mathcal{L}}_{\pi}$. Clearly, $\varepsilon_{\pi}=\rho_{\pi}^{-1}$. A lower bound on $\rho_{\pi}$ is now given. Note that $\hat{\mathcal{L}}_{\pi}^{\dagger}$ is obtained by changing the sign of the first term in (27). Multiplying out, it follows that

$$
\hat{\mathcal{L}}_{\pi}^{\dagger} \hat{\mathcal{L}}_{\pi}=a^{2} I_{\pi}+a D_{\pi}+S_{\pi}+D_{\pi}^{2}
$$

where $S_{\pi}$ is equal to

$$
-\sum_{i, j=1}^{d} b_{i} b_{j} X_{i}^{\pi} X_{j}^{\pi}+\sum_{i, j, k=1}^{d} b_{k} c_{i j}\left(X_{i}^{\pi} X_{j}^{\pi} X_{k}^{\pi}-X_{k}^{\pi} X_{j}^{\pi} X_{i}^{\pi}\right) .
$$

Using the fact that the $X_{i}^{\pi}$ are skew Hermitian, it can be shown $S_{\pi}$ is Hermitian positive definite. From (28), $\rho_{\pi}$ is larger than the smallest eigenvalue of $D_{\pi}^{2}$. By (27) and (101), if $\delta_{\pi}$ is an eigenvalue of $D_{\pi}$ then $\left|\delta_{\pi}\right| \geq \gamma_{1} \kappa_{\pi}$. Thus, $\rho_{\pi} \geq \gamma_{1}^{2} \kappa_{\pi}^{2}$. The proposition follows immediately. 
It is now possible to obtain a family of linear matrix equations equivalent to (1). Recall, $\mathcal{L} u$ is interpreted as in (8). Thus, $\mathcal{L} u \in$ $D^{\prime}(G)$ for $u \in D^{\prime}(G)$. By an application of (8) and (12)

$$
\widehat{\mathcal{L} u}(\pi)=\hat{\mathcal{L}}_{\pi} \hat{u}(\pi)
$$

so that (1) is equivalent to

$$
\hat{\mathcal{L}}_{\pi} \hat{u}(\pi)=\hat{\omega}(\pi) .
$$

The existence, uniqueness, and regularity of the solution of the (1) are obtained in the following proposition.

Proposition 3 [Solution of (1)]: Let $\omega \in D^{\prime}(G)$ so that $\omega \in$ $H_{n}(G)$ for some $n \in \mathbb{Z}$. Equation (1) has a unique solution $u \in H_{n+2}(G)$ and $u$ is given by

$$
\hat{u}(\pi)=\hat{\mathcal{L}}_{\pi}^{-1} \hat{\omega}(\pi) .
$$

In particular, $\omega \in D(G)$ implies $u \in D(G)$ and $\omega \in H_{n}(G)$, where $n>d / 2$ implies $u$ has a modification in $C^{2}(G)$.

Proof: It has been noted that (1) is equivalent to (30). If a solution $u$ exists, then its uniqueness follows from the fact that $\hat{\mathcal{L}}_{\pi}$ is invertible, which was stated in Proposition 2. To show that a solution exists, it is enough to prove that $u$ given by (31) indeed belongs to $H_{n+2}(G)$.

Note that (31) implies

$$
\operatorname{tr}\left\{\hat{u}_{\pi} \hat{u}_{\pi}^{\dagger}\right\} \leq \varepsilon_{\pi} \operatorname{tr}\left\{\hat{\omega}_{\pi} \hat{\omega}_{\pi}^{\dagger}\right\}
$$

where $\varepsilon_{\pi}$ is given in Proposition 2. Applying the result of Proposition 2 , it is possible to evaluate (14)

$$
\begin{aligned}
& \|u\|_{n+2}^{2} \leq\left(a^{-1} \hat{\omega}\left(\pi_{0}\right)\right)^{2}+ \\
& \alpha \sum_{\pi \neq \pi_{0}} d_{\pi}\left(\frac{1+\kappa_{\pi}}{\kappa_{\pi}}\right)^{2}\left(1+\kappa_{\pi}\right)^{n} \operatorname{tr}\left\{\hat{\omega}_{\pi} \hat{\omega}_{\pi}^{\dagger}\right\} .
\end{aligned}
$$

Let $\kappa_{1}$ be the first nonzero element of the sequence $\left(\kappa_{\pi}\right)$. That is, $\kappa_{1}$ is the spectral gap of the Laplace operator $\Delta$. The following estimate is straightforward:

$$
\|u\|_{n+2}^{2} \leq\left(a^{-1} \hat{\omega}\left(\pi_{0}\right)\right)^{2}+\alpha\left(\frac{1+\kappa_{1}}{\kappa_{1}}\right)^{2}\|\omega\|_{n}^{2} .
$$

In particular, $\|u\|_{n+2}<\infty$ and $u \in H_{n+2}(G)$.

That $u$ is indeed a solution follows by replacing (31) in (30). Finally, assume $\omega \in D(G)$. Then, by (17), $\omega \in H_{n}(G)$ for all $n \in \mathbb{N}$. By the result just proved, it also follows $u \in H_{n}(G)$ for all $n \in \mathbb{N}$. Then, (17) implies $u \in D(G)$. Similarly, if $\omega \in H_{n}(G)$ where $n>d / 2$, then $u \in H_{2+n}(G)$, and since $2+n>2+d / 2$, the Sobolev embedding theorem implies $u$ has a modification in $C^{2}(G)$.

To write down the solution of (1) in the form of a convolution, it is enough to compare (25) and (31). The question arises of whether there exists a distribution $e \in D^{\prime}(G)$ such that

$$
\hat{e}(\pi)=\hat{\mathcal{L}}_{\pi}^{-1} \quad \pi \in \hat{G} .
$$

The affirmative answer to this question is a result of Proposition 3. In fact, (31) implies that (33) gives the unique solution of

$$
\mathcal{L} e=\delta .
$$

Indeed, applying (12) to $\delta$ gives

$$
\hat{\delta}(\pi)=I_{\pi} \quad \pi \in \hat{G} .
$$

Thus, $e$ verifies (30) with $\omega=\delta$. Moreover, it is clear from (25) and (31) that the solution of (1) is given by

$$
u=e * \omega
$$

This justifies the name fundamental solution for $e$. In order to understand the regularity of $e$, note the following recent result of Applebaum [14]:

$$
n>d / 2 \Rightarrow \sum_{\pi} d_{\pi}^{2}\left(1+\kappa_{\pi}\right)^{-n}<\infty .
$$

From (14) and Proposition 3, it then follows

$$
n>d / 2 \Rightarrow \delta \in H_{-n}(G) \text { and } e \in H_{2-n}(G) .
$$

In particular, if $d=3$ as is the case for $G=S O(3)$, then $e \in L^{2}(G)$. This example is considered in Section V.

\section{STATIONARY FIELDS AND DISTRIBUTIONS}

WSS random fields were studied by Yaglom and have a theory similar to that of WSS processes. The main ingredients in this theory are the covariance function and its spectral representation [7]. Here, Section III-A recovers Yaglom's theory and Section III-B generalizes it to the study of random distributions. In particular, the notion of WSS random distribution is introduced. A WSS random distribution has a "covariance function" which is in fact a distribution. In Section IV, this is applied to the concept of white noise indexed on $G$, which turns out to be a WSS distribution whose covariance is the Dirac delta. In Section III-C, a necessary and sufficient condition is given for the existence of derivatives in the square mean of WSS random fields. This is helpful in understanding PDE which involve WSS random fields.

\section{A. Stationary Random Fields}

The aim is now to reformulate the theory of WSS random fields in a way which generalizes immediately to random distributions. Assume given a probability space $(\Omega, \mathcal{A}, \mathbb{P})$ and write $\mathbb{E}$ for expectation. Let $L^{2}(\Omega \times G)$ be the space of measurable mappings $\xi: \Omega \times G \rightarrow \mathbb{C}$ such that the random variable $\xi(g)$ where $\xi(g)(\omega)=\xi(\omega, g)$ verifies $\mathbb{E} \xi(g)=0$ and $\mathbb{E}|\xi(g)|^{2}<\infty$ for almost all $g \in G$ and such that, moreover,

$$
\int \mathbb{E}|\xi(g)|^{2} d g<\infty .
$$

In particular, (39) implies the random function $\xi$ where $\xi(\omega)(g)=\xi(\omega, g)$ is almost surely in $L^{2}(G)$. Thus, there exist random variables $\hat{\xi}(\pi)$ for $\pi \in \hat{G}$ such that

$$
\xi=\sum_{\pi} d_{\pi} \operatorname{tr}\left\{\hat{\xi}(\pi) U^{\pi}\right\}
$$

where the series converges in $L^{2}(G)$ almost surely. Also

$$
\mathbb{E}\|\xi\|^{2}=\sum_{\pi} d_{\pi} \operatorname{tr}\{b(\pi)\}<\infty
$$


where

$$
b(\pi)=\mathbb{E}\left\{\hat{\xi}(\pi) \hat{\xi}^{\dagger}(\pi)\right\} .
$$

Of course, (40) and (41) follow from (90) and (91). Conversely, given any random variables $\hat{\xi}(\pi)$ which verify (41), there exists a unique $\xi \in L^{2}(\Omega \times G)$ verifying (40). Here, uniqueness is understood up to almost sure equality in $L^{2}(G)$. An element $\xi \in L^{2}(\Omega \times G)$ is referred to as a random field on $G$.

In order to introduce the covariance function of $\xi$, the following notation is used. Let $\beta(g)$ be given by

$$
\beta(g)=\int \xi(h g) \xi^{*}(h) d h .
$$

Note that this can be written

$$
\beta(g)=(\xi * \tilde{\xi})(g)
$$

where $\tilde{\xi}(g)=\xi^{*}\left(g^{-1}\right)$. Since the convolution of two functions in $L^{2}(G)$ is again in $L^{2}(G)$, it follows that $\beta \in L^{2}(G)$ almost surely. By applying (21) and doing a direct calculation

$$
\hat{\beta}(\pi)=\hat{\xi}(\pi) \hat{\xi}^{\dagger}(\pi) .
$$

Indeed, transforming $\xi$ into $\tilde{\xi}$ amounts to taking the Hermitian transpose of each $\hat{\xi}(\pi)$.

It follows from (39) and (42), by the Cauchy-Schwarz inequality, that $\mathbb{E} \beta(g)$ is finite. This is noted $\mathbb{E} \beta(g)=B(g)$. Now, $B$ is bounded, so that it is possible to find $\hat{B}(\pi)$ for $\pi \in \hat{G}$. These are then given by

$$
\hat{B}(\pi)=b(\pi) .
$$

The function $B$ is called the covariance function of $\xi$.

A strong result is that $B$ has a continuous modification, for any $\xi \in L^{2}(\Omega \times G)$. This is shown by noting the Fourier series of $B$ converges absolutely and uniformly [15].

The random field $\xi \in L^{2}(\Omega \times G)$ is called WSS if for all $g_{1}$, $g_{2}, h \in G$

$$
\mathbb{E} \xi\left(g_{1}\right) \xi^{*}\left(g_{2}\right)=\mathbb{E} \xi\left(h g_{1}\right) \xi^{*}\left(h g_{2}\right) .
$$

In this case, the definition of $B$ gives in (42)

$$
B(g)=\int \mathbb{E} \xi(h g) \xi^{*}(h) d h=\mathbb{E} \xi(g) \xi^{*}(1) .
$$

Thus, in the case where $\xi$ is WSS, the definition (46) implies

$$
B(g)=\mathbb{E} \xi(h g) \xi^{*}(h)
$$

for the covariance function $B$.

In [7], Yaglom proved the following statement.

Theorem 2 (Characterization of WSS Fields): Assume $\xi \in$ $L^{2}(\Omega \times G)$ with $\hat{\xi}(\pi)$ and $b(\pi)$ given as in (40) and (41). Then, $\xi$ is WSS iff

$$
d_{\pi} \mathbb{E} \hat{\xi}_{j i}(\pi) \hat{\xi}_{l k}^{*}\left(\pi^{\prime}\right)=\delta_{\pi \pi^{\prime}} \delta_{i k} b_{j l}(\pi) .
$$

When $\xi$ is WSS, this theorem allows the random variable $\xi(g)$ to be identified with the corresponding sum in (40).

$$
\xi(g)=\sum_{\pi} d_{\pi} \operatorname{tr}\left\{\hat{\xi}(\pi) U^{\pi}(g)\right\} .
$$

Indeed, (41) and (48) imply that this sum converges in $L^{2}(\Omega)$. As stated after (41), the sum in the right-hand side defines a random function which is almost surely equal to $\xi$ in $L^{2}(G)$. Making this identification, the function $B$ can be computed for every $g \in G$

$$
B(g)=\mathbb{E}\left[\sum_{\pi} d_{\pi} \operatorname{tr}\left\{\hat{\xi}(\pi) U^{\pi}(g)\right\} \sum_{\pi^{\prime}} d_{\pi^{\prime}} \operatorname{tr}\left\{\hat{\xi}\left(\pi^{\prime}\right)\right\}\right] .
$$

Since both sums converge in $L^{2}(\Omega)$, (48) gives

$$
B(g)=\sum_{\pi} d_{\pi} \operatorname{tr}\left\{b(\pi) U^{\pi}(g)\right\} .
$$

In other words, $B$ is equal to the pointwise sum of its Fourier series. This last result has the following corollaries. First, $B$ is continuous. Indeed, it has been stated the Fourier series of $B$ converges absolutely and uniformly. Second, $\xi$ is continuous in the square mean. This is since

$$
\mathbb{E}|\xi(h g)-\xi(h)|^{2}=2 \Re\{B(e)-B(h)\} .
$$

This result on continuity in the square mean is complemented by the result on the existence of derivatives in the square mean, given in Section III-C.

In order to motivate the introduction of WSS random distributions in Section III-B, note the following property of WSS random fields. If $\xi \in L^{2}(\Omega \times G)$ is WSS, then for all $k_{1}$, $k_{2} \in D(G)$

$$
\begin{aligned}
\mathbb{E}\left\langle k_{1}, \xi\right\rangle\left\langle\xi, k_{2}\right\rangle & =\iint k_{1}(h) k_{2}^{*}(g) \mathbb{E} \xi(g) \xi^{*}(h) d h d g \\
& =\iint k_{1}(h) k_{2}^{*}(g) B\left(h^{-1} g\right) d h d g \\
& =\iint k_{1}\left(g h^{-1}\right) B(h) k_{2}^{*}(g) d h d g
\end{aligned}
$$

where the last equality uses the invariance of the Haar measure under group operations. By comparing to (20), it is possible to write

$$
\mathbb{E}\left\langle k_{1}, \xi\right\rangle\left\langle\xi, k_{2}\right\rangle=\left\langle B * k_{1}, k_{2}\right\rangle .
$$

This property will be used to define WSS random distributions, as it can be rewritten using the notation of (5).

\section{B. Stationary Random Distributions}

WSS random distributions will arise as the solutions of white noise PDE. In order to introduce them, it will be suitable to consider the space $D^{\prime}(\Omega \times G)$. This is the space of mappings $u: \Omega \times D(G) \rightarrow \mathbb{C}$ with the following properties. For $\omega \in \Omega$, the functional $u(\omega): D(G) \rightarrow \mathbb{C}$, given by $u(\omega)(k)=u(\omega, k)$ for $k \in D(G)$, belongs to $D^{\prime}(G)$. Moreover, for $k \in D(G)$, the random variable $u(k)$, given by $u(k)(\omega)=u(\omega, k)$, belongs to $L^{2}(\Omega)$.

Using the notation of (5), the space $L^{2}(\Omega \times G)$ is identified with a subspace of $D^{\prime}(\Omega \times G)$. For $\xi \in L^{2}(\Omega \times G)$, its regularity properties can be characterized using random Sobolev spaces. This is addressed in Section III-C. 
Let $u \in D^{\prime}(\Omega \times G)$. For $\pi \in \hat{G}$, it is possible to define

$$
b(\pi)=\mathbb{E}\left\{\hat{u}(\pi) \hat{u}^{\dagger}(\pi)\right\}
$$

where $\hat{u}(\pi)$ is given by (12). The expectation is finite since, by hypothesis, $u\left(U_{i j}^{\pi}\right) \in L^{2}(\Omega)$ for $i, j=1, \ldots, d_{\pi}$. For $n \in \mathbb{Z}$, $H_{n}(\Omega \times G)$ is defined as the space of $u \in D^{\prime}(\Omega \times G)$ such that

$$
\left.\|u\|\right|_{n} ^{2}=\sum_{\pi} d_{\pi}\left(1+\kappa_{\pi}\right)^{n} \operatorname{tr}\{b(\pi)\}<\infty .
$$

When $u \in H_{n}(\Omega \times G)$, it is clear from (52) that $u$ verifies (14) almost surely and

$$
\left.\|u\|\right|_{n} ^{2}=\mathbb{E}\|u\|_{n}^{2}
$$

This norm arises from an inner product, which is obtained by taking the expectation of the inner product in $H_{n}(G)$. If random variables $\hat{u}(\pi)$ exist which verify the condition (52) then these define a unique $u \in H_{n}(\Omega \times G)$.

Moreover, if $u_{1} \in H_{-n}(\Omega \times G)$ and $u_{2} \in H_{n}(\Omega \times G)$,

$$
\mathbb{E}\left|u_{1}\left(u_{2}\right)\right| \leq\left|\left\|u_{1}\right\|\right|_{-n}\left|\left\|u_{2}\right\|\right|_{n} .
$$

This follows since $u_{1} \in H_{-n}(G)$ and $u_{2} \in H_{n}(G)$ almost surely. Then, by (15)

$$
\left|u_{1}\left(u_{2}\right)\right| \leq\left\|u_{1}\right\|_{-n}\left\|u_{2}\right\|_{n}
$$

and it is enough to take expectations and apply the Cauchy-Schwarz inequality (in $L^{2}(\Omega)$ ). In fact, it is possible to show $H_{-n}(\Omega \times G)$ is the dual space of $H_{n}(\Omega \times G)$ according to norm (53).

A random distribution $u \in D^{\prime}(\Omega \times G)$ is called integrable if there exists a distribution $\mathbb{E} u \in D^{\prime}(G)$ such that

$$
\mathbb{E} u(k)=\mathbb{E}\{u(k)\} \quad k \in D(G) .
$$

Naturally, $\mathbb{E} u$ is referred to as the expectation of $u$. A straightforward result is that if $u \in H_{-n}(\Omega \times G)$, for some $n \in \mathbb{N}$, then $u$ is integrable and $\mathbb{E} u \in H_{-n}(G)$. Indeed, for $f \in H_{n}(\Omega \times G)$, (54) implies

$$
|\mathbb{E} u(f)| \leq \mathbb{E}|u(f)| \leq|\|u\||_{-n}\|f\|_{n} .
$$

This shows $\mathbb{E} u$ is continuous on $H_{n}(G)$. Thus, $\mathbb{E} u \in H_{-n}(G)$.

Now, the covariance of a random distribution $u \in D^{\prime}(\Omega \times G)$ can be introduced using a formula similar to (43). For this, it is required $u \in H_{-n}(\Omega \times G)$ for some $n \in \mathbb{N}$.

Let $\tilde{u} \in D^{\prime}(\Omega \times G)$ be given by

$$
\tilde{u}(k)^{*}=u(\tilde{k}) \quad k \in D(G) .
$$

Here, $\tilde{k} \in D(G)$ is given by $\tilde{k}(g)=k^{*}\left(g^{-1}\right)$. Formally, repeating (43), let

$$
\beta=u * \tilde{u} .
$$

A direct calculation using (12) and (25) shows

$$
\hat{\beta}(\pi)=\hat{u}(\pi) \hat{u}^{\dagger}(\pi) .
$$

Similar to (44). The covariance $B$ of $u$ is to be defined by $B=$ $\mathbb{E} \beta$. It is now shown that if $u \in H_{-n}(\Omega \times G)$ then $B$ is well defined with $B \in H_{-2 n}(G)$. For this, it is enough to prove there exists $B \in H_{-2 n}(G)$ such that

$$
\hat{B}(\pi)=b(\pi) .
$$

Indeed, noting $b(\pi)=\hat{\beta}(\pi)$ by (51) and (58), it then follows from (13) that $B$ and $\beta$ verify (55).

In order to prove $B$ given by (59) exists, it is enough to check condition (14). Note first that since $b(\pi)$ is Hermitian positive definite

$$
\operatorname{tr}\{b(\pi) b(\pi)\} \leq \operatorname{tr}\{b(\pi)\} \operatorname{tr}\{b(\pi)\} .
$$

Replacing in (14) gives

$$
\|B\|_{-2 n} \leq \mid\|u\|_{-n} \sup _{\pi}\left(1+\kappa_{\pi}\right)^{-n} \operatorname{tr}\{b(\pi)\}
$$

which is finite by (52), whenever $u \in H_{-n}(\Omega \times G)$.

It is now possible to define WSS random distributions by generalizing (50). A random distribution $u \in H_{-n}(\Omega \times G)$, where $n \in \mathbb{N}$, will be called WSS if

i) $\mathbb{E} u=0$

ii) For all $k_{1}, k_{2} \in D(G)$,

$$
\mathbb{E} u\left(k_{1}\right) u\left(k_{2}\right)^{*}=\left\langle B * k_{1}, k_{2}\right\rangle .
$$

This leads to a characterization similar to Yaglom's result of Theorem 2.

Theorem 3 (Characterization of WSS Distributions): Assume $u \in H_{-n}(\Omega \times G)$ where $n \in \mathbb{N}$, with $\hat{u}(\pi)$ and $b(\pi)$ given as in (12) and (51). Then, $u$ is WSS iff $\mathbb{E} \hat{u}(\pi)=0$ and

$$
d_{\pi} \mathbb{E} \hat{u}_{j i}(\pi) \hat{u}_{l k}^{*}\left(\pi^{\prime}\right)=\delta_{\pi \pi^{\prime}} \delta_{i k} b_{j l}(\pi) .
$$

Proof: Note that $u$ is integrable and $\mathbb{E} u \in H_{-n}(G)$, since $u \in H_{-n}(\Omega \times G)$. By (13), condition i) of the definition is equivalent to $\hat{u}(\pi)=0$. Thus, it is enough to show (60) is equivalent to (61).

Assume (60) holds and replace

$$
k_{1}=U_{k l}^{\pi} \quad k_{2}=U_{i j}^{\pi^{\prime}} .
$$

These are clearly in $D(G)$ and verify, for $\pi^{\prime \prime} \in \hat{G}$,

$$
\begin{aligned}
& \hat{k}_{1, p m}\left(\pi^{\prime \prime}\right)=d_{\pi}^{-1} \delta_{\pi \pi^{\prime \prime}} \delta_{p l} \delta_{m k} \\
& \hat{k}_{2, p m}\left(\pi^{\prime \prime}\right)=d_{\pi^{\prime}}^{-1} \delta_{\pi^{\prime} \pi^{\prime \prime}} \delta_{p j} \delta_{m i} .
\end{aligned}
$$

Applying (60) and using (23) and (91), it follows the left-hand side of (61) is equal to

$$
\delta_{\pi \pi^{\prime}} \sum_{r, p, m=1}^{d_{\pi}} b_{r \cdot p}(\pi) \delta_{p l} \delta_{m k} \delta_{m i} \delta_{r j}=\delta_{\pi \pi^{\prime}} \delta_{i k} b_{j l}(\pi)
$$

which is the right-hand side. 
Conversely, assume (61) holds. By (19) and (52), the following sums converge in $L^{2}(\Omega)$ for all $k_{1}, k_{2} \in D(G)$ :

$$
\begin{gathered}
u\left(k_{1}\right)=\sum_{\pi} d_{\pi} \operatorname{tr}\left\{\hat{k}_{1}(\pi) \hat{u}^{\dagger}(\pi)\right\} \\
u\left(k_{2}\right)^{*}=\sum_{\pi} d_{\pi^{\prime}} \operatorname{tr}\left\{\hat{u}\left(\pi^{\prime}\right) \hat{k}_{2}^{\dagger}\left(\pi^{\prime}\right)\right\} .
\end{gathered}
$$

Thus, it is possible to multiply term by term and apply (61). Terms with $\pi \neq \pi^{\prime}$ disappear, so the left-hand side of (60) is equal to

$$
\sum_{\pi} d_{\pi} \sum_{j, i=1}^{d_{\pi}} \sum_{l, k=1}^{d_{\pi}} \hat{k}_{2, j i}^{*}(\pi) \hat{k}_{1, l k}(\pi) \delta_{i k} b_{j l}(\pi) .
$$

This is seen to be equal to

$$
\sum_{\pi} d_{\pi} \operatorname{tr}\left\{b(\pi) \hat{k}_{1}(\pi) \hat{k}_{2}^{\dagger}(\pi)\right\}
$$

by (23) and (91), it follows that (60) holds.

Before going on, note the following property which will be used in Section IV. Similar results, in the special case of random fields, play an important role in [5] and [6].

Proposition 4 (Convolution Preserves WSS): Let $\phi \in$ $H_{m}(G)$ and $u \in H_{n}(\Omega \times G)$ where $n, m \in \mathbb{Z}$. Then, $\phi * u \in H_{n+m}(\Omega \times G)$. Moreover, if $u$ is WSS, then $\phi * u$ is WSS with covariance

$$
B^{\phi}=\phi * B * \tilde{\phi}
$$

where $B$ is the covariance of $u$.

Proof: That $\phi * u \in H_{n+m}(\Omega \times G)$ can be checked from condition (52). Indeed, letting $u_{\phi}=\phi * u$, using (23)

$$
\begin{aligned}
& \sum_{\pi} d_{\pi}\left(1+\kappa_{\pi}\right)^{n+m} \mathbb{E} \operatorname{tr}\left\{\hat{u}_{\phi}(\pi) \hat{u}_{\phi}^{\dagger}(\pi)\right\} \leq \\
& \sum_{\pi} d_{\pi}\left(1+\kappa_{\pi}\right)^{n+m} \operatorname{tr}\left\{\hat{\phi}(\pi) \hat{\phi}^{\dagger}(\pi)\right\} \operatorname{tr}\{b(\pi)\} \leq \\
& |\|u\||_{n} \sup _{\pi}\left(1+\kappa_{\pi}\right)^{m} \operatorname{tr}\left\{\hat{\phi}(\pi) \hat{\phi}^{\dagger}(\pi)\right\} .
\end{aligned}
$$

The norm of $u$ is finite by hypothesis. Similarly, the supremum is finite since $\phi$ satisfies (14) for $m$. This shows that (52) holds.

If $u$ is WSS, then $\phi * u$ can be proved to be WSS using (61) of Theorem 3. This can be checked easily so it will not be detailed here.

To obtain the covariance $B^{\phi}$ of $u_{\phi}$, note that

$$
\hat{u}_{\phi}(\pi) \hat{u}_{\phi}^{\dagger}(\pi)=\hat{\phi}(\pi) \hat{u}(\pi) \hat{u}^{\dagger}(\pi) \hat{\phi}^{\dagger}(\pi) .
$$

By (51) and (59)

$$
\hat{B}^{\phi}(\pi)=\hat{\phi}(\pi) b(\pi) \hat{\phi}^{\dagger}(\pi) .
$$

That $B$ verifies (62) now follows from (25).

\section{Derivatives in the Square Mean}

The formalism of random Sobolev spaces allows the classical notion of derivative in the square mean to be recovered.

Consider a random field $\xi \in L^{2}(\Omega \times G)$ and assume moreover $\xi \in H_{n}(\Omega \times G)$ for some $n \in \mathbb{N}-\{0\}$. This implies that $\xi \in$ $H_{n}(G)$ almost surely. In other words, $\xi$ has weak derivatives $X^{\beta} \xi$ for all $|\beta| \leq n$ which verify $X^{\beta} \xi \in L^{2}(G)$ almost surely. The following proposition summarizes some properties of these weak derivatives.

Proposition 5 (Weak Derivatives): Assume $\xi \in H_{n}(\Omega \times G)$ for some $n \in \mathbb{N}-\{0\}$. The weak derivatives $X^{\beta} \xi$ for $|\beta| \leq n$ verify $X^{\beta} \xi \in L^{2}(\Omega \times G)$. Moreover, if $\xi$ is WSS, then $X^{\beta} \xi$ is WSS.

Proof: The proof considers the case $n=1$. The general case can be obtained by induction. Assume then $\xi \in H_{1}(\Omega \times G)$. By writing (7) in integral form, it is possible to find

$$
\int X_{i} \xi(g) k(g) d g=-\int \xi(g) X_{i} k(g) d g \quad k \in D(G)
$$

for $i=1, \ldots, d$. Taking the expectation of both sides and using the fact that $\mathbb{E} \xi(g)=0$ for almost all $g \in G$, it follows that

$$
\int \mathbb{E}\left\{X_{i} \xi(g)\right\} k(g) d g=0, \quad k \in D(G) .
$$

This implies $\mathbb{E}\left\{X_{i} \xi(g)\right\}=0$ for almost all $g \in G$.

Note that by $(96)$

$$
\widehat{X_{i} \xi}(\pi)=X_{i}^{\pi} \hat{\xi}(\pi) .
$$

To show $X_{i} \xi \in L^{2}(\Omega \times G)$ it remains to check

$$
\sum_{\pi} d_{\pi} \operatorname{tr}\left\{b^{i}(\pi)\right\}<\infty
$$

where

$$
b^{i}(\pi)=-X_{i}^{\pi} b(\pi) X_{i}^{\pi} .
$$

Indeed, by (41) this would imply (39) holds for $X_{i} \xi$.

Note, since $b(\pi)$ is Hermitian positive definite

$$
\operatorname{tr}\left\{b^{i}(\pi)\right\} \leq-\operatorname{tr}\left\{X_{i}^{\pi} X_{i}^{\pi}\right\} \operatorname{tr}\{b(\pi)\} .
$$

Using the fact that each $X_{i}^{\pi}$ is skew Hermitian, this further implies by (101)

$$
\operatorname{tr}\left\{b^{i}(\pi)\right\} \leq \kappa_{\pi} \operatorname{tr}\{b(\pi)\} .
$$

Since $\xi$ satisfies (52), it is easy to conclude

$$
\sum_{\pi} d_{\pi} \operatorname{tr}\left\{b^{i}(\pi)\right\} \leq \mid\|\xi\|_{1}
$$

which proves $X_{i} \xi \in L^{2}(\Omega \times G)$. Now, any $X \xi$ where $X \in \mathfrak{g}$ is a linear combination of $X_{i} \xi$.

For the second claim, if $\xi$ is WSS, then using (96) as above it straightforward to show $X \xi$ verifies the condition (48) of Theorem 2. 
An interesting formula follows from the second part of this proposition. If $\xi$ is WSS, then

$$
\mathbb{E} X_{i} \xi(g) X_{j} \xi^{*}(h)=-X_{j(h)} X_{i(g)} B\left(h^{-1} g\right) .
$$

Here, $X_{j(h)}$ means that $X_{j}$ acts on the variable $h$ and similarly for $X_{i(g)}$. This formula is a clear generalization of a classical one. In order to prove it, note that $X \xi$ is WSS so that, as in (49),

$$
X \xi(g)=\sum_{\pi} d_{\pi} \operatorname{tr}\left\{X^{\pi} \hat{\xi}(\pi) U^{\pi}(g)\right\}
$$

for $X \in \mathfrak{g}$, where the series converge in $L^{2}(\Omega)$. Two series of this form, for $X_{i} \xi(g)$ and $X_{j} \xi^{*}(h)$, can be multiplied term by term and the expectation (64) obtained using (48).

When $\xi$ is WSS, the weak derivatives $X^{\beta} \xi$ are moreover derivatives in the square mean. Here, the derivative in the square mean of $\xi$ at $g \in G$ in the direction of $X$ is the limit in $L^{2}(\Omega)$

$$
\lim _{t \rightarrow 0} \frac{\xi\left(g e^{t X}\right)-\xi(g)}{t}
$$

when it exists. Note the usual notation for the exponential application from $\mathfrak{g}$ onto $G$ is used.

For simplicity, the theorem is only stated for first derivatives, $X \xi$ where $X \in \mathfrak{g}$.

Theorem 4 (Derivatives in the Square Mean): Assume $\xi \in$ $L^{2}(\Omega \times G)$ is WSS. Then, $\xi \in H_{1}(\Omega \times G)$ iff for $g \in G$ the limit (66) exists and is equal to the sum (65).

Proof: Assume $\xi \in H_{1}(\Omega \times G)$. Since $\xi$ is WSS, $X \xi$ is WSS by Proposition 5. It follows, as already noted, that $X \xi(g)$ can be identified with the sum (65) for $g \in G$.

By (49), the difference between the expression under the limit in (66) and $X \xi(g)$ is equal to

$$
\sum_{\pi} d_{\pi} \operatorname{tr}\left\{X^{\pi}(t) \hat{\xi}(\pi) U^{\pi}(g)\right\}
$$

where, exp being the matrix exponential,

$$
X^{\pi}=(1 / t)\left\{\exp \left(t X^{\pi}\right)-I_{\pi}-X^{\pi}\right\} .
$$

Using (48), the square mean of (67) is equal to

$$
\sum_{\pi} d_{\pi} \operatorname{tr}\left\{b(\pi) X^{\pi}(t) X^{\pi \dagger}(t)\right\} .
$$

Now, it is clear that $X^{\pi}(t) \rightarrow 0$ as $t \rightarrow 0$. Thus, to show that the square mean of (67) also converges to 0 as $t \rightarrow 0$, it is enough to bound (68) independently of $t$.

Note that since $b(\pi)$ is positive definite

$$
\operatorname{tr}\left\{b(\pi) X^{\pi}(t) X^{\pi \dagger}(t)\right\} \leq \operatorname{tr}\{b(\pi)\}\left|X^{\pi}(t) X^{\pi \dagger}(t)\right|_{\mathrm{op}}
$$

where $\left|X^{\pi}(t) X^{\pi \dagger}(t)\right|_{\text {op }}$ denotes the Euclidean operator norm.

The formula for the Taylor remainder of the first-order development of the matrix exponential gives

$$
\left|X^{\pi}(t)\right|_{\mathrm{op}} \leq 2\left|X^{\pi}\right|_{\mathrm{op}} .
$$

Let $X=x_{1} X_{1}+\cdots+x_{d} X_{d}$. It now follows by (69) and (101) $\left|X^{\pi}(t) X^{\pi \dagger}(t)\right|_{\mathrm{op}}=\left|X^{\pi}(t)\right|_{\mathrm{op}}^{2} \leq-4 \operatorname{tr}\left\{X^{\pi} X^{\pi}\right\} \leq 4|X|^{2} \kappa_{\pi}$ where $|X|^{2}=x_{1}^{2}+\cdots+x_{d}^{2}$. Returning to (68), the sum therein is found to be less of equal to

$$
4|X|^{2} \sum_{\pi} d_{\pi} \kappa_{\pi} \operatorname{tr}\{b(\pi)\} \leq \mid\|\xi\|_{1}
$$

which is independent of $t$.

For the if part, assume the sum (65) converges in $L^{2}(\Omega)$ for $X \in \mathfrak{g}$ and $g \in G$. Since $\xi$ is WSS, the square mean of this sum can be calculated using (48). In particular, replacing $X=X_{i}$ gives

$$
\mathbb{E}\left|\sum_{\pi} d_{\pi} \operatorname{tr}\left\{X^{\pi} \hat{\xi}(\pi) U^{\pi}(g)\right\}\right|^{2}=-\sum_{\pi} d_{\pi} \operatorname{tr}\left\{X_{i}^{\pi} X_{i}^{\pi} b(\pi)\right\} .
$$

This is finite by assumption, so summing over $i=1, \ldots, d$ gives by (101)

$$
\sum_{\pi} d_{\pi} \kappa_{\pi} \operatorname{tr}\{b(\pi)\}<\infty .
$$

Since $\xi \in L^{2}(\Omega \times G)$ it follows $\xi \in H_{1}(\Omega \times G)$.

\section{White Noise ElLIPTIC EQUATION}

Building on the results of the previous sections, a stochastic version of (1) can be studied. This is written as follows

$$
\mathcal{L} u=w
$$

where now $w \in D^{\prime}(\Omega \times G)$ is a random distribution.

Note that for each realization of the right-hand side $w$, the stochastic (70) reduces to an instance of the deterministic (1). This can be solved as in Section II-B, using (36) and the random solution $u$ obtained in this way satisfies Proposition 3 .

The aim should then be to find suitable conditions under which $u$ belongs to $D^{\prime}(\Omega \times G)$. It is shown in Proposition 6 that $w \in H_{n}(\Omega \times G)$ implies $u \in H_{n+2}(\Omega \times G)$, for $n \in \mathbb{Z}$.

A further question relates to the stationarity of $u$. In general, it is possible to show that if $w$ is a WSS random distribution as in Section III-B then so is $u$. It is more interesting to study this question when $w$ is a special kind of WSS random distribution; namely, a white noise distribution indexed on $G$. This is introduced in the following theorem.

Theorem 5 (White Noise): Let $n>d / 2$. There exists $w \in$ $H_{-n}(\Omega \times G)$ which is WSS with covariance $B^{w}=\delta$. In particular, $w$ verifies the isometry property

$$
\mathbb{E} w\left(k_{1}\right) w\left(k_{2}\right)^{*}=\left\langle k_{1}, k_{2}\right\rangle
$$

for $k_{1}, k_{2} \in D(G)$.

Proof: If a WSS $w \in H_{-n}(\Omega \times G)$ is shown to exist with $B^{w}=\delta$, then (71) follows from (60). Indeed, by (6) and (22), $\delta * k_{1}=k_{1}$ for $k_{1} \in D(G)$. To show that $w$ exists, let $\hat{w}(\pi)$ for $\pi \in \hat{G}$ be given by

$$
\begin{gathered}
\mathbb{E} \hat{w}(\pi)=0 \\
d_{\pi} \mathbb{E} \hat{w}_{j i}(\pi) \hat{w}_{l k}^{*}\left(\pi^{\prime}\right)=\delta_{\pi \pi^{\prime}} \delta_{i k} \delta_{j l} .
\end{gathered}
$$

Then

$$
b^{w}(\pi) \equiv \mathbb{E} \hat{w}(\pi) \hat{w}^{\dagger}(\pi)=I_{\pi} .
$$


Using (37), it is shown that condition (52) holds, so that $w \in$ $H_{-n}(\Omega \times G)$. That $w$ is WSS is an immediate result of (61).

To see that the covariance $B^{w}$ is equal to $\delta$, note that (59) and (74) imply

$$
\hat{B}(\pi)=I_{\pi}
$$

which is the same as (35).

In the framework based on Section III-B, it is meaningless to speak of the value $w(g)$ of a white noise $w$ at $g \in G$. However, the isometry property (71) can be obtained formally using the notation $w(g)$. The result that $w$ is WSS and $B^{w}=\delta$ seems to suggest

$$
\mathbb{E} w(g) w^{*}(h)=\delta\left(h^{-1} g\right)
$$

Formally

$$
\begin{aligned}
\mathbb{E} w\left(k_{1}\right) w\left(k_{2}\right)^{*} & =\iint k_{1}(g) k_{2}^{*}(h) \mathbb{E} w(g) w^{*}(h) d g d h \\
& =\iint k_{1}(g) k_{2}^{*}(h) \delta\left(h^{-1} g\right) d g d h \\
& =\iint k_{1}(g) k_{2}^{*}(g) d g
\end{aligned}
$$

which is the same as (71).

Proposition 6 relates the properties of the right-hand side $w$ to those of the random solution $u$ in (70).

Proposition 6 [Solution of (70)]: For $w \in H_{n}(\Omega \times G)$, with $n \in \mathbb{Z},(70)$ has a unique solution $u \in H_{n+2}(\Omega \times G)$. Moreover, if $w$ is WSS with covariance $B^{w}$, then $u$ is WSS with covariance $B=e * B^{w} * \tilde{e}$.

Proof: Assume $w \in H_{n}(\Omega \times G)$, with $n \in \mathbb{Z}$. From (31)

$$
\hat{u}(\pi)=\hat{\mathcal{L}}_{\pi}^{-1} \hat{w}(\pi)
$$

In order to check that $u$ verifies condition (52) for $n+2$, it is possible to proceed as in the proof of Proposition 3.

Clearly

$$
b(\pi)=\hat{\mathcal{L}}_{\pi}^{-1} b^{w}(\pi) \hat{\mathcal{L}}_{\pi}^{-1 \dagger}
$$

Since $b^{w}(\pi)$ is positive definite

$$
\operatorname{tr}\{b(\pi)\} \leq \varepsilon_{\pi} \operatorname{tr}\left\{b^{w}(\pi)\right\}
$$

where $\varepsilon_{\pi}$ is given in Proposition 2 .

Replacing this inequality in condition (52) gives

$$
\begin{aligned}
& \left.\|u\|\right|_{n+2} ^{2} \leq\left(a^{-1} b^{w}\left(\pi_{0}\right)\right)^{2}+ \\
& \alpha \sum_{\pi \neq \pi_{0}} d_{\pi}\left(\frac{1+\kappa_{\pi}}{\kappa_{\pi}}\right)^{2}\left(1+\kappa_{\pi}\right)^{n} \operatorname{tr}\left\{b^{w}(\pi)\right\} .
\end{aligned}
$$

Let $\kappa_{1}$ be the spectral gap of the Laplace operator $\Delta$. The following estimate is straightforward:

$$
\left.\|u\|\right|_{n+2} ^{2} \leq\left(a^{-1} b^{w}\left(\pi_{0}\right)\right)^{2}+\alpha\left(\frac{1+\kappa_{1}}{\kappa_{1}}\right)^{2} \mid\|w\|_{n}^{2} .
$$

Since $w \in H_{n}(\Omega \times G)$, this implies $u \in H_{n+2}(\Omega \times G)$.

Recall formula (36)

$$
u=e * w .
$$

This formula along with Proposition 4 provides the second part of the current proposition.

It is interesting to consider the above Proposition when $w$ is a white noise indexed on $G$. In this case, the solution $u$ has covariance $B=e * \tilde{e}$. Then

$$
b(\pi)=\hat{e}(\pi) \hat{e}^{\dagger}(\pi)
$$

which follows by (63) and Theorem 5 .

If the operator $\mathcal{L}$ is sufficiently symmetric, then $e$ can be recovered from the above formula. Indeed, if $b_{i}=0$ in the expression (2), then (27) and (33) imply $\hat{e}(\pi)$ is Hermitian strictly positive definite. Then, $\hat{e}(\pi)$ is simply the Hermitian square root of $b(\pi)$. In this case, if $e$ depends on some unknown parameters, then it is possible to attempt to identify these parameters given $b(\pi)$. More generally, by obtaining a sufficient number of Fourier coefficients $\hat{e}(\pi)$, an approximation of $e$ may be constructed.

\section{EXample: A White Noise PDE on SO(3)}

Here, a concrete example is given of the development in the previous section. This example illustrates Theorem 5 and Proposition 6.

The example treated will consider random fields arising from a white noise PDE defined on the special orthogonal group $S O(3)$. This is the group of real $3 \times 3$ matrices $g$ which satisfy

$$
g g^{T}=I, \quad \operatorname{det}(g)=1
$$

where ${ }^{T}$ denotes the transpose. In other words, $g \in S O(3)$ is a unit determinant orthogonal matrix. The group operation is matrix multiplication. The identity element, here noted $I$, is the $3 \times 3$ identity matrix and $g^{-1}$ is the matrix inverse of $g$, equal to its transpose.

The white noise PDE to be studied is the Hemholtz equation on $S O(3)$. This is the equation

$$
(\Delta-\beta) u=w
$$

where $\beta \geq 0$ is the "wave number," $w$ is a spatial white noise on $S O(3)$, and $\Delta$ is the Laplace operator.

To describe these more precisely, it is necessary to recall some facts from harmonic analysis on $S O(3)$. For these, the reader is referred to [16].

The Lie algebra $\mathfrak{s o}(3)$ of $S O(3)$ is of dimension $d=3$. It is well known that $\mathfrak{s} \mathfrak{o}(3)$ can be identified with the space of $3 \times 3$ real antisymmetric matrices. This is done in the following way.

Consider the matrices $x_{1}, x_{2}, x_{3}$ with matrix elements

$$
x_{i, k j}=\epsilon_{i k j}
$$

where $\epsilon_{i k j}$ is totally antisymmetric with $\epsilon_{123}=-1$. These form a basis of the space of $3 \times 3$ antisymmetric matrices. The Lie algebra $\mathfrak{s o}(3)$ of $S O(3)$ has basis $X_{1}, X_{2}, X_{3}$ where for $f \in$ $C^{1}(S O(3))$

$$
X_{i} f(g)=\left.\frac{d}{d t}\right|_{t=0} f\left(g \exp \left(t x_{i}\right)\right)
$$

which is a result of (84). 
Irreducible representations of $S O(3)$ are usually indexed by their highest weights, $l \in \mathbb{N}$. To each value of $l$, there corresponds an irreducible representation with unitary representative $U^{l}$.

These can be obtained by recursively forming tensor products. For $l=0, U^{l}$ is the trivial representation of dimension $d_{0}=1$. The corresponding eigenvalue of the Laplace operator is $\kappa_{0}=0$. Since $S O(3)$ is connected, this is a simple eigenvalue.

The first nontrivial representation corresponds to $l=1$. This is the identity representation

$$
U^{1}(g)=g
$$

this acts as a unitary transformation of $\mathbb{R}^{3}$, (i.e., it preserves Euclidean scalar product). Clearly, $d_{1}=3$. A straightforward substitution shows the matrices $X_{i}^{1}$ arising as in (93) are $X_{i}^{1}=$ $x_{i}$. Applying (101), it follows $\kappa_{1}=2$.

It can be shown by induction the dimension of the representation of weight $l$ is $2 l+1$ and $\kappa_{l}=l(l+1)$. Assume $U^{l}$ is given, for $l \geq 1$, and satisfies these relations.

To obtain $U^{l+1}$, it is possible to form the tensor product

$$
U=U^{l} \otimes U^{1}
$$

This defines a unitary representation of $S O(3)$ which has dimension $(2 l+1) \times 3$, the product of the dimensions of $U^{l}$ and $U^{1}$. The Clebsch-Gordan formula asserts $U$ can be transformed, by a change of basis, into the direct sum

$$
U=U^{l-1} \oplus U^{l} \oplus U^{l+1}
$$

By subtracting the dimensions, it follows $d_{l+1}=2 l+3$. This is $2(l+1)+1$, so that by induction the dimension formula holds. In a similar way, after applying (101), the formula for $\kappa_{l}$ follows by induction.

The above decomposition allows not only for $d_{l}$ and $\kappa_{l}$ to be calculated, but even for the matrix elements of $U^{l}(g)$ to be expressed as homogeneous polynomials in those of $g$. Thus, an analytic expression of the $U^{l}$ is obtained. However, much more often, $U^{l}$ is given in terms of Wigner $D$-functions of the Euler angles of $g$.

Assuming the $U^{l}$ are given, applying (27) and (33), the fundamental solution of (79) is given by

$$
\hat{e}(l)=\frac{-1}{\beta+\kappa_{l}} I_{l}
$$

where $I_{l}$ is the $2 l+1 \times 2 l+1$ identity matrix.

Based on (38), since $d=3$ it follows $e \in L^{2}(S O(3))$. In other words, $e$ can be identified with a square integrable function, with respect to the Haar measure of $S O(3)$. This will again be noted $e$.

This square integrable function can be given in terms of the probability density of the Laplace probability distribution described in [17]. An analytic expression of $e$ is then found based on [18]. Thus, for the current example, the fundamental solution is a square integrable function and has a closed-form expression.
Now, according to (78), the covariance $B$ of $u$ has Fourier coefficients

$$
b(l)=\left(\beta+\kappa_{l}\right)^{-2} I_{l} .
$$

Since $e \in L^{2}(S O(3))$ and $B=e * \tilde{e}$, Proposition 1 shows $B \in L^{2}(S O(3))$. Moreover, $e \in L^{2}(S O(3))$ also implies that $u$ verifies condition (41).

Thus $u$ can be constructed pointwise as in (49)

$$
u(g)=\sum_{l}(2 l+1) \operatorname{tr}\left\{\hat{u}(l) U^{l}(g)\right\}
$$

where by (48) and (81), the random variables $\hat{u}(l)$ verify

$$
\mathbb{E} \hat{u}_{j i}(l) \hat{u}_{l k}^{*}\left(l^{\prime}\right)=\delta_{l l^{\prime}} \delta_{i k} \delta_{j l}\left(\beta+\kappa_{l}\right)^{-2} .
$$

The last formula gives a possible numerical construction of the value of $u$ at a given point $g \in S O(3)$. Indeed, this can be approximated in the square mean by generating random variables $\hat{u}(l)$ with the indicated covariance structure and summing sufficiently many terms in (82). model

\section{CONCLUSION}

This paper is meant as a step toward a more concrete study of wide sense stationary random fields defined on groups. Precisely, the aim is to introduce these random fields not immediately through their definition but to construct them as solutions of white noise PDEs. This has been realized in the special case of compact Lie groups, although at the price of a generalization which considers random distributions instead of random fields. This section aims for an informal discussion and will not make the distinction between fields and distributions.

There are several advantages to the construction of wide sense stationary random fields as solutions of white noise PDEs. First, PDEs are well-known models of many physical phenomena. Thus, random fields arising in applied problems may naturally obey PDEs where white noise represents ambient environmental fluctuations.

Second, random fields which satisfy the white noise PDE studied above [see (70)] belong to a parametric family given by the coefficients of the differential operator. This can also be extended to depend on an additional parameter which is the order of the differential operator, here restricted to be of order two. The existence of sufficiently general parametric models allows a more robust approach to modeling, where parameters can be fitted to data. Also, the existence of such parametric models allows for pertinent estimation and statistical decision problems to be posed.

Third, invariant white noise PDEs follow the well-known paradigm of systems theory. If the solution is represented as a convolution with the fundamental solution [see (77)], then clearly $w$ appears as the input and $u$ the output of a linear invariant system. For these systems, there is the open perspective of formulating control or observation problems. These can be based on the existing literature for control of PDEs in Euclidean space [19].

Still, there exist important examples of wide sense stationary random fields which do not clearly satisfy a white noise PDE. 
Above all, this is the case for fractional Brownian fields on the sphere (equivalently, the rotation group) [20].

The model considered here contains many limitations and is far from exhausting the class of wide sense stationary random fields on Lie groups. There is the restriction to compact Lie groups, which is a matter of technical convenience. Also, the model was limited to second-order elliptic operators. This restriction can be dropped by considering higher order elliptic or semielliptic operators. It is also possible to consider parabolic equations which represent time dependent random fields, the simplest example being a stochastic heat equation. These parabolic equations can display both space and time stationarity.

It is important to note that the computer simulation of random fields or of PDEs implies considerable difficulties. These are due to complexity as well as to the high volume of data which needs to be processed. For a square integrable random field with a given covariance function, a comprehensive approach to simulation is proposed in [21].

The study of random fields defined on Lie groups may seem unnatural since Lie groups mostly appear as groups of transformations of some underlying homogeneous space. In this situation, one would be more interested in random fields defined on the homogeneous space and which are wide sense stationary under the action of the Lie group of transformations. It should be noted that any object (i.e., function, distribution or measure) defined on a homogeneous space can be lifted in a canonical way to an object defined on its Lie group of transformations. Thus, after some minor modifications, the framework proposed in this paper can be used to study random fields defined on homogeneous spaces. For example, random fields defined on a sphere can be considered as a special case of random fields defined on the special orthogonal group.

\section{APPENDIX A \\ HARMONIC ANALYSIS ON COMPACT LIE GROUPS}

The aim of this appendix is to recover the background material in harmonic analysis on compact Lie groups which is used in the body of the paper. The fundamental theorem for harmonic analysis on compact Lie groups is the Peter-Weyl theorem, Theorem 6 below. This is here presented along with some additional results on differential and integral calculus. For a very clear presentation of this material, the book of Faraut is recommended [22].

Let $G$ be a compact connected Lie group with identity 1 and Lie algebra $\mathfrak{g}$. The elements of $\mathfrak{g}$ are identified with left invariant vector fields on $G$. Various spaces of complex-valued functions on $G$ may be introduced. For example, $C(G), C^{k}(G)$, and $C^{\infty}(G)$ are the spaces of continuous, $k$ times continuously differentiable and infinitely differentiable functions from $G$ to C.

A definition of left invariant vector fields can be given based on the group exponential mapping. For each $X \in \mathfrak{g}$, there exists a unique one parameter subgroup, $\gamma: \mathbb{R} \rightarrow G$. This is defined by

$$
\dot{\gamma}(t)=X(\gamma(t)) \quad \gamma(t+s)=\gamma(t) \gamma(s)
$$

Here, the dot denotes differentiation with respect to $t$. Since there is a clear resemblance to the usual definition of the exponential, it is common to write

$$
\gamma(t)=e^{t X} .
$$

For $f \in C^{1}(G)$, a function $X f \in C(G)$ is defined by

$$
X f(g)=\left.\frac{d}{d t}\right|_{t=0} f\left(g e^{t X}\right) .
$$

The operation taking $f$ to $X f$ is a first-order differential operator. That is, it is linear and verifies the product rule, $X\left(f_{1} f_{2}\right)=$ $f_{2} X f_{1}+f_{1} X f_{2}$ for $f_{1}, f_{2} \in C^{1}(G)$. The name left invariant reflects the fact that $X$, when seen as a differential operator, commutes with left translations. For $g \in G$, the left translation $L_{g}: G \rightarrow G$ is the mapping $L_{g}(h)=g h$. Now, it is easy to see in (84)

$$
X\left(f \circ L_{g}\right)=(X f) \circ L_{g}
$$

for any $g \in G$. Given two left invariant vector fields $X, Y \in \mathfrak{g}$, it can be shown there exists a further $Z \in \mathfrak{g}$ such that

$$
Z f=X Y f-Y X f
$$

for $f \in C^{2}(G)$ and where $X Y f$ means successive application of $Y$ and then $X$ to $f$. The left invariant vector field $Z$ is denoted $[X, Y]$ and known as the Lie bracket of $X$ and $Y$.

The space $L^{2}(G)$ consists of functions which are square integrable with respect to the Haar measure. After identification of functions which are modifications of each other, i.e., which differ only on a set of measure 0 , this is a Hilbert space with inner product

$$
\left\langle f_{1}, f_{2}\right\rangle=\int f_{1}(g) f_{2}^{*}(g) d g
$$

where ${ }^{*}$ denotes complex conjugation and the integral is extended over $G$ with respect to the Haar measure $d g$. The norm corresponding to this inner product is denoted $\|f\|$ for $f \in$ $L^{2}(G)$.

The dual of $G$ is denoted $\hat{G}$. This is the set of equivalence classes of irreducible representations of $G$. For $\pi \in \hat{G}$, its dimension is denoted $d_{\pi}$ and it is assumed a smooth unitary representative $U^{\pi}$ has been chosen. Thus, $U^{\pi}: G \rightarrow S U\left(d_{\pi}\right)$ is a homomorphism from $G$ to the matrix group $S U\left(d_{\pi}\right)$ of unit determinant unitary $d_{\pi} \times d_{\pi}$ matrices. That is, for $g, h \in G$ and $X \in \mathfrak{g}$,

$$
U^{\pi}(g h)=U^{\pi}(g) U^{\pi}(h) \quad U^{\pi}\left(e^{t X}\right)=\exp \left(t X^{\pi}\right) .
$$

Here, the product on the right-hand side of the first identity is a matrix product. The exponential exp in the second identity is a usual matrix exponential. The matrix $X^{\pi}$ is given by

$$
X^{\pi}=\left.\frac{d}{d t}\right|_{t=0} U^{\pi}\left(e^{t X}\right)
$$

and it is clear that $X^{\pi}$ is skew Hermitian; indeed it is the derivative of a special unitary matrix. Incidentally, definition (86) gives

$$
[X, Y]^{\pi}=X^{\pi} Y^{\pi}-Y^{\pi} X^{\pi} .
$$


The trivial representation is denoted $\pi_{0} \in \hat{G}$. This is characterized by $d_{\pi_{0}}=1$. Throughout this paper, whenever a sum $\sum_{\pi}$ appears, it should be extended over all $\pi \in \hat{G}$. Note also, expressions of the form $X U^{\pi}$ and $\int U^{\pi}$ simply mean differentiation or integration of each element of the matrix $U^{\pi}$.

Theorem 6 (Peter-Weyl Theorem): Let $f \in L^{2}(G)$ and define its Fourier coefficient matrices by

$$
\hat{f}(\pi)=\int f(g) U^{\pi}\left(g^{-1}\right) d g
$$

for $\pi \in \hat{G}$. The following Fourier series converges in $L^{2}(G)$ :

$$
f=\sum_{\pi} d_{\pi} \operatorname{tr}\left\{\hat{f}(\pi) U^{\pi}\right\}
$$

Moreover, the following Plancherel formula holds:

$$
\left\langle f_{1}, f_{2}\right\rangle=\sum_{\pi} d_{\pi} \operatorname{tr}\left\{\hat{f}_{1}(\pi) \hat{f}_{2}^{\dagger}(\pi)\right\}
$$

for $f_{1}, f_{2} \in L^{2}(G)$, where ${ }^{\dagger}$ denotes the Hermitian transpose. Finally, if $f \in C(G)$, then the Fourier series (90) converges uniformly to $f$.

The underlying fact for the Peter-Weyl theorem is that the matrix elements $U_{i j}^{\pi}$ where $\pi \in \hat{G}$ and $i, j=1, \ldots, d_{\pi}$ give an orthonormal basis of $L^{2}(G)$

$$
\left\langle U_{j i}^{\pi}, U_{l k}^{\pi^{\prime}}\right\rangle=d_{\pi} \delta_{\pi \pi^{\prime}} \delta_{i k} \delta_{j l}
$$

where the notation on the right-hand side is for the Kronecker delta.

Fourier series such as (90) can be used to represent the derivatives of $f$, in case $f \in C^{k}(G)$. For $X \in \mathfrak{g}$ and $\pi \in \hat{G}$, it follows from (84) and (88) that

$$
X U^{\pi}=U^{\pi} X^{\pi} .
$$

In order to apply this note that for $f_{1}, f_{2} \in C^{1}(G)$

$$
\left\langle X f_{1}, f_{2}\right\rangle=-\left\langle f_{1}, X f_{2}\right\rangle .
$$

Further application of this formula gives

$$
\left\langle X Y f_{1}, f_{2}\right\rangle=\left\langle f_{1}, Y X f_{2}\right\rangle=-\left\langle Y f_{1}, X f_{2}\right\rangle
$$

for $f \in C^{2}(G)$. In fact, (94) follows easily if one notes

$$
\int X|f(g)|^{2} d g=0
$$

and $X|f|^{2}=X f f^{*}+f X f^{*}$. That the above integral is zero expresses the right invariance of the Haar measure. That is

$$
\int|f(g)|^{2} d g=\int\left|f\left(g e^{t X}\right)\right|^{2} d g
$$

for each $t \in \mathbb{R}$, so that the above derivative should be zero.

Using (89), (93) and (94), (95), it follows that

$$
\begin{gathered}
\widehat{X f}(\pi)=X^{\pi} \hat{f}(\pi), \quad f \in C^{1}(G) \\
\widehat{X Y} f(\pi)=X^{\pi} Y^{\pi} \hat{f}(\pi), \quad f \in C^{2}(G) .
\end{gathered}
$$

The most important differential operator on $G$ is the Laplace operator $\Delta$. In order to define this, let $(\cdot, \cdot)$ be an Ad-invariant scalar product of $\mathfrak{g}$ and $X_{1}, \ldots, X_{d}$ a corresponding orthonormal basis. The property of Ad-invariance means that for $X, Y, Z \in \mathfrak{g}$

$$
([X, Y], Z)+(Y,[X, Z])=0 .
$$

In other words, the Lie bracket operation is antisymmetric. Once such a scalar product has been chosen, the Laplace operator is defined to be

$$
\Delta=\sum_{i=1}^{d} X_{i} X_{i}
$$

It follows from (95) that $\Delta$ is negative. That is, for $f \in C^{2}(G)$

$$
\langle\Delta f, f\rangle=-\sum_{i=1}^{d}\left\|X_{i} f\right\|^{2} .
$$

Characteristic of compact Lie groups is the fact that $\Delta$ is diagonalized in the orthonormal basis described in (92). That is

$$
\Delta U^{\pi}=-\kappa_{\pi} U^{\pi} \kappa_{\pi} \geq 0 .
$$

Since $G$ is connected, $\kappa_{\pi_{0}}=0$ is a simple eigenvalue. That is, $\kappa_{\pi}>0$ for $\pi \neq \pi_{0}$. This can also be expressed by saying that $\Delta f=0$ implies $f=\bar{f}$ where

$$
\bar{f} \equiv \int f(g) d g=\hat{f}\left(\pi_{0}\right) .
$$

Note from (97) that (100) is equivalent to

$$
\sum_{i=1}^{d} X_{i}^{\pi} X_{i}^{\pi}=-\kappa_{\pi} .
$$

In connection with the definition of Sobolev spaces in Section II-A, note that it is possible to combine (91), (99) and (101) to write

$$
\sum_{i=1}^{d}\left\|X_{i} f\right\|^{2}=\sum_{\pi} d_{\pi} \kappa_{\pi} \operatorname{tr}\left\{\hat{f}(\pi) \hat{f}^{\dagger}(\pi)\right\} .
$$

The eigenvalues $\left(\kappa_{\pi} ; \pi \in \hat{G}\right)$ can be arranged in a sequence $\kappa_{\pi} \uparrow \infty$. By default, sums over $\pi \in \hat{G}$ are carried out in the same order.

The following matrix inequalities which are useful in the paper. Let $A$ be a Hermitian positive-definite matrix and $X$ some other matrix, with both being square and of the same dimension. Then

$$
\operatorname{tr}\{A X\} \leq \operatorname{tr}\{A\}|X|_{\mathrm{op}}
$$

where $|X|_{\mathrm{op}}$ denotes the Euclidean operator norm. If $X$ is also Hermitian positive definite, e.g., $X=A$, then

$$
\operatorname{tr}\{A X\} \leq \operatorname{tr}\{A\} \operatorname{tr}\{X\} .
$$

Indeed, in this case, $|X|_{\text {op }}$ is less than the trace of $X$. Inequality (103) can be found on Page 169 of [15]. It follows from the statement that the trace of $A$ is the norm of the linear form which 
to each $X$ associates the left-hand side of the inequality. That is,

$$
\operatorname{tr}\{A\}=\sup \left\{\operatorname{tr}\{A X\} ;|X|_{\text {op }} \leq 1\right\} .
$$

In the body of the paper, these two inequalities are considered as known and are used repeatedly.

Let $B: G \rightarrow \mathbb{C}$ be a bounded function and note $\hat{B}(\pi)=b(\pi)$ for $\pi \in \hat{G}$. Assume $B$ is positive definite. That is, $B$ verifies

$$
\iint k(g) B\left(h^{-1} g\right) k^{*}(h) d h d g \geq 0
$$

for all $k \in C^{\infty}(G)$.

In [15], it is shown $B$ is positive definite iff $b(\pi)$ is Hermitian positive definite, for $\pi \in \hat{G}$. The Fourier series of a positivedefinite function converges absolutely and uniformly. From the definition of positive definiteness, it follows that

$$
\sum_{\pi} d_{\pi} \operatorname{tr}\{b(\pi)\}<\infty .
$$

Applying (103) in the Fourier series (90) of $B$ gives

$$
\sum_{\pi} d_{\pi}\left|\operatorname{tr}\left\{b(\pi) U^{\pi}(g)\right\}\right| \leq \sum_{\pi} d_{\pi} \operatorname{tr}\{b(\pi)\} .
$$

This is since, $U^{\pi}(g)$ being unitary, $\left|U^{\pi}(g)\right|_{\mathrm{op}}=1$. The absolute and uniform convergence follow immediately. These imply the Fourier series of $B$ is pointwise summable. Since it converges uniformly, its sum is a continuous function. In particular, if $B$ is equal to the pointwise sum of its Fourier series, then $B$ is continuous. These results are used in Section III, (see the discussion of (45) therein).

Conversely, it is interesting to note that a continuous function $B$ is positive definite iff

$$
\sum_{i, j=1}^{m} c_{i} c_{j}^{*} B\left(g_{j}^{-1} g_{i}\right) \geq 0
$$

for all $c_{1}, \ldots, c_{m} \in \mathbb{C}$ and $g_{1}, \ldots, g_{m} \in G$, where $m$ is any positive integer.

Positive-definite functions are among the fundamental objects of harmonic analysis on compact Lie groups. It is easy to see positive-definite functions $B$ which satisfy $B(1)=c$, for some fixed $c \in \mathbb{R}$, form a convex set. This observation can be used as a starting point to the whole study of irreducible representations and may even extend to the case where the underlying group is noncompact.

\section{APPENDIX B}

\section{B-Sobolev SPaCes on the CiRCle}

In Section II-A, Sobolev spaces $H_{n}(G)$ for $n \in \mathbb{Z}$ were introduced. These appear as subspaces of the space of distributions $D^{\prime}(G)$ with a hierarchy given by the Sobolev embedding theorem, Theorem 1.

Throughout this paper, Sobolev spaces were mostly studied using Harmonic analysis. This is based on the simple relations (13) and (14), which can be thought of as operative definitions.
The aim of this appendix is to present an elementary example of the discussion in Section II-A. For this, consider $G=S^{1}$ the unit circle group. This is the very simplest compact Lie group. It is the subset $S^{1} \subset \mathbb{C}$ consisting of those complex numbers $g$ such that $|g|=1$, considered with the operation of multiplication of complex numbers.

All $g \in S^{1}$ can be written $g=e^{i \theta}$ where $\theta \in \mathbb{R}$. Accordingly, a function $f: S^{1} \rightarrow \mathbb{C}$ can be identified with a function $F$ : $\mathbb{R} \rightarrow \mathbb{C}$ which is $2 \pi$-periodic

$$
F(\theta)=f\left(e^{i \theta}\right) .
$$

Various spaces of functions $C\left(S^{1}\right), C^{k}\left(S^{1}\right)$ and $C^{\infty}\left(S^{1}\right)$ are then identified with the spaces of $2 \pi$-periodic functions $F$ of the above form which are in $C(\mathbb{R}), C^{k}(\mathbb{R})$ and $C^{\infty}(\mathbb{R})$ respectively. It is natural, and often useful, to identify $f$ and $F$ and write $f(\theta)$ instead of $f(g)$. This is here done.

The dual of $S^{1}$ is $\hat{S}^{1}=\mathbb{Z}$. For $l \in \mathbb{Z}$, the corresponding unitary representative is given by

$$
U^{l}\left(e^{i \theta}\right)=e^{i l \theta} .
$$

This is a homomorphism from $S^{1}$ to the matrix group $S U(1)$, which is isomorphic to $S^{1}$.

The Peter-Weyl theorem (Theorem 6) here reduces to the statement that the Fourier series of any function $f \in L^{2}\left(S^{1}\right)$ converges in $L^{2}\left(S^{1}\right)$ and the same Fourier series of any function $f \in C\left(S^{1}\right)$ converges uniformly. This is just the classical Fourier series

$$
f(\theta)=\sum_{l \in \mathbb{Z}} \hat{f}(l) e^{i l \theta}
$$

with Fourier coefficients

$$
\hat{f}(l)=\frac{1}{2 \pi} \int_{0}^{2 \pi} f(\theta) e^{-i l \theta} .
$$

When $f$ is not continuous, it is an abuse of notation to include the argument $\theta$ in the above expression for the Fourier expansion. Indeed, convergence does not hold pointwise.

The rate of convergence of the Fourier series of a function $f \in$ $L^{2}\left(S^{1}\right)$ is related to the regularity (differentiability) properties of $f$. This relation naturally leads to the introduction of Sobolev spaces $H_{n}\left(S^{1}\right)$ for $n \in \mathbb{Z}$.

Recall for $f \in L^{2}\left(S^{1}\right)$, the Plancherel formula holds

$$
\|f\|=\sum_{l \in \mathbb{Z}}|\hat{f}(l)|^{2}<+\infty .
$$

The Laplace operator $\Delta$ on $S^{1}$ is given by

$$
\Delta f=\frac{d^{2} f}{d \theta^{2}} f \in C^{2}\left(S^{1}\right) .
$$

Its eigenvalues are $-l^{2}$ for $l \in \mathbb{Z}$ with eigenfunctions given from

$$
\frac{d^{2} e^{i l \theta}}{d \theta^{2}}=-l^{2} e^{i l \theta} .
$$

For $n \in \mathbb{N}$, the Sobolev space $H_{n}\left(S^{1}\right)$ is the dense subspace of $L^{2}\left(S^{1}\right)$ consisting of those functions $f$ such that

$$
\sum_{l \in \mathbb{Z}} l^{2 n}|\hat{f}(l)|^{2}<+\infty .
$$


Since $f \in L^{2}\left(S^{1}\right)$, so it verifies the Plancherel formula, this is equivalent to

$$
\sum_{l \in \mathbb{Z}}\left(1+l^{2}\right)^{n}|\hat{f}(l)|^{2}<+\infty .
$$

When this is verified, the following Fourier series converges in $L^{2}\left(S^{1}\right)$ :

$$
f^{(n)}(\theta)=\sum_{l \in \mathbb{Z}} l^{n} \hat{f}(l) e^{i l \theta}
$$

and $f^{(n)} \in L^{2}\left(S^{1}\right)$ is the weak derivative, of order $n$, of $f$.

The Sobolev space $H_{-n}\left(S^{1}\right)$, where $n \in \mathbb{N}$, is the dual space of $H_{n}\left(S^{1}\right)$. It consists of those distributions $u \in D^{\prime}\left(S^{1}\right)$ such that

$$
\sum_{l \in \mathbb{Z}}\left(1+l^{2}\right)^{-n}|\hat{u}(l)|^{2}<+\infty
$$

where $\hat{u}(l)$ is the Fourier coefficient

$$
\hat{u}(l)=u\left(U^{l}\right) .
$$

The duality between $H_{-n}\left(S^{1}\right)$ and $H_{n}\left(S^{1}\right)$, where $n \in \mathbb{N}$, is given by

$$
u(f)=\sum_{l \in \mathbb{Z}} \hat{f}(l) \hat{u}^{*}(l)
$$

for $f \in H_{n}\left(S^{1}\right)$ and $u \in H_{-n}\left(S^{1}\right)$. This implies that there exists a function $u_{(n)} \in L^{2}\left(S^{1}\right)$ such that

$$
u(f)=\frac{\hat{u}(0)}{2 \pi} \int_{0}^{2 \pi} f(\theta) d \theta+\frac{1}{2 \pi} \int_{0}^{2 \pi} f^{(n)}(\theta) u_{(n)}^{*}(\theta) d \theta .
$$

For example, let $\delta$ be the Dirac delta, so that by definition $\delta(f)=f(0)$ for $f \in D\left(S^{1}\right)$; note that $D\left(S^{1}\right)$ is the same set as $C^{\infty}\left(S^{1}\right)$. Then, $\hat{\delta}(l)=1$ for $l \in \mathbb{Z}$ and it follows that

$$
\sum_{l \in \mathbb{Z}}\left(1+l^{2}\right)^{-1}|\hat{\delta}(l)|^{2}<+\infty
$$

which is equivalent to $\delta \in H_{-1}\left(S^{1}\right)$. For $f \in H_{1}\left(S^{1}\right)$

$$
\delta(f)=\frac{1}{2 \pi} \int_{0}^{2 \pi} f(\theta) d \theta+\frac{1}{2 \pi} \int_{0}^{2 \pi} f^{(1)}(\theta) \delta_{(1)}^{*}(\theta) d \theta
$$

where $\delta_{(1)} \in L^{2}\left(S^{1}\right)$ is given by

$$
\delta_{(1)}(\theta)=\sum_{l \in \mathbb{Z}} l^{-1} e^{i l \theta}
$$

which can be thought of as the "reproducing kernel" on the Hilbert space $H_{1}\left(S^{1}\right)$.

The most important idea to keep in mind, in preparation for the discussion of Section II-A, is the relation between the rate of convergence of the Fourier series of a function $f$ and the regularity of this function. This relation is quantified by introducing Sobolev spaces which turn out to be the natural tool for the problem studied in this paper.

\section{REFERENCES}

[1] A. Yaglom, An Introduction to the Theory of Stationary Random Functions. New York, NY, USA: Dover Phoenix Edition, 2004.

[2] R. Azencott and D. Dacunha-Castelle, Séries D'observations Irrégulières, Modélisation et Prévision. Paris, France: Masson, 1984.

[3] L. Schwartz, Mathematics for the Physical Sciences. New York, NY, USA: Addison-Wesley, 1966.

[4] H. Holden, B. Oksendal, J. Uboe, and T. Zhang, Stochastic Partial Differential Equations, A Modeling White Noise Functional Approach, 2nd ed. New York, NY, USA: Springer-Verlag, 2010.

[5] B. Yazici, "Stochastic deconvolution over groups," IEEE Trans. Inf. Theory, vol. 50, no. 3, pp. 494-510, Mar. 2004.

[6] H. Arora and R. Parthasarathy, "Optimal estimation and detection in homogeneous spaces," IEEE Trans. Signal Process., vol. 58, no. 5, pp. 2623-2635, May 2010.

[7] A. M. Yaglom, "Second order homogeneous random fields," in Proc. 4th Berkley Symp. Math. Statist. Probab., 1961, vol. 2, pp. 593-622.

[8] J. Koo and P. T. Kim, "Asymptotic minimax bounds for stochastic deconvolution over groups," IEEE Trans. Inf. Theory, vol. 54, no. 1, pp. 198-289, Jan. 2008.

[9] S. Said, C. Lageman, N. L. Bihan, and J. H. Manton, "Decompounding on compact Lie groups," IEEE Trans. Inf. Theory, vol. 56, no. 6, pp. 2766-2777, Jun. 2010.

[10] S. Said and J. H. Manton, "Extrinsic mean of Brownian distributions on compact Lie groups," IEEE Trans. Inf. Theory, vol. 58, no. 6, pp. 3521-3535, Jun. 2012.

[11] M. Ruzhansky and V. Turunen, Pseudo-Differential Operators and Symmetries: Background Analysis and Advanced Topics. Basel, Switzerland: Birkhäuser, 2009.

[12] H. Brezis, Functional Analysis, Sobolev Spaces and Partial Differential Equations, 1st ed. New York, NY, USA: Springer-Verlag, 2010.

[13] G. S. Chirikjian and A. B. Kyatkin, Engineering Applications of Noncommutative Harmonic Analysis. Boca Raton, FL, USA: CRC Press, 2000.

[14] D. Applebaum, "Infinitely divisible central probability measures on compact Lie groups-Regularity, semigroups and transition kernels," Ann. Probabil., vol. 39, no. 6, pp. 2474-2496, 2011.

[15] R. Edwards, Integration and Harmonic Analysis on Compact Groups. Cambridge, U.K.: Cambridge Univ. Press, 1972.

[16] S. L. Altmann, Rotations, Quaternions and Double Groups. New York, NY, USA: Dover, 2005.

[17] J. Koo and P. T. Kim, "Optimal spherical deconvolution," J. Multivariate Anal., vol. 80, no. 1, pp. 21-42, 2002.

[18] D. M. Healy, H. Hendriks, and P. T. Kim, "Spherical deconvolution," J. Multivariate Anal., vol. 67, no. 1, pp. 1-22, 1998.

[19] F. Troltzsch, Optimal Control of Partial Differential Equations. Providence, RI, USA: Amer. Math. Soc., 2010.

[20] J. Istas, "Karhunen-Loève expansion of spherical fractional Brownian motion," Statist. Probabil. Lett., vol. 76, pp. 1578-1583, 2006.

[21] A. Brouste, J. Istas, and S. Lambert-Lacroix, "Statistical inference on stationary random fields," J. Statist. Softw., vol. 36, no. 4, pp. 1-14, 2010.

[22] J. Faraut, Analysis on Lie Groups, an Introduction. Cambridge, U.K.: Cambridge Univ. Press, 2008.

Salem Said completed his Ph.D. at Institut Polytechnique de Grenoble in 2009 , under the supervision of Drs. Nicolas Le Bihan and Stephen J. Sangwine. His Ph.D. theses was titled "Estimation and filtering of processes in matrix Lie groups". Since 2010 he has been working at the Department of Electrical and Electronic Engineering of the University of Melbourne, under the supervision of Pr. Jonathan H. Manton. His research focuses on stochastic processes with values in manifolds, related estimation and filtering problems and their application to wave physics. His broad area of expertise is in stochastic calculus and its application to signal processing and statistics. 
Pierre-Olivier Amblard was born in Dijon, France, in 1967. He received the Ingénieur degree in electrical engineering in 1990 from Ecole Nationale Supérieure des Ingénieurs Electriciens de Grenoble, Institut National Polytechnique de Grenoble. He received the DEA (M.Sc.) in signal processing and the Doctorat (Ph.D.) in 1990 and 1994 respectively, both from the INPG. He received the Habilitation á Diriger des Recherches in 2001. Since 1994, he works for the Centre National de la Recherche Scientifique (CNRS) where he is a Directeur de Recherche. He is with GIPSAlab, Grenoble, France, in the team CICS (Communication and Information in Complex Systems), but is currently on secondment in the Department of Mathematics and Statistics of the University of Melbourne. His research interests include statistical signal processing, non-stationary signal processing, applications of signal processing in physics and neuroscience.
Jonathan H. Manton (M'02-SM'03) received the B.Sc. degree in mathematics and the B.Eng. degree in electrical engineering in 1995 and the Ph.D. degree in 1998, all from the University of Melbourne, Melbourne, Vic., Australia. He holds a Distinguished Chair at the University of Melbourne with the title Future Generation Professor. He is also an Adjunct Professor in the Mathematical Sciences Institute at the Australian National University. From 1998 to 2004, he was with the Department of Electrical and Electronic Engineering at the University of Melbourne. During that time, he held a Postdoctoral Research Fellowship then subsequently a Queen Elizabeth II Fellowship, both from the Australian Research Council. In 2005 he became a Full Professor in the Department of Information Engineering, Research School of Information Sciences and Engineering (RSISE), Australian National University. From July 2006 to May 2008 , he was on secondment to the Australian Research Council as Executive Director, Mathematics, Information, and Communication Sciences. His traditional research interests range from pure mathematics (e.g., commutative algebra, algebraic geometry, differential geometry) to engineering (e.g., signal processing, wireless communications). Recently though, led by a desire to participate in the convergence of the life sciences and the mathematical sciences, he has started to apply his expertise to research in neuroscience. He also has extensive experience in software development. Prof. Manton has served recently as an Associate Editor for the IEEE TRANSACTIONS ON Signal PROCESSING, a Committee Member for the IEEE Signal Processing for Communications (SPCOM) Technical Committee, and a Committee Member on the Mathematics Panel for the ACT Board of Senior Secondary Studies in Australia. He is currently on the Editorial Board of Systems and Control Letters. He has recently received a 2009 Future Summit Australian Leadership Award in recognition of outstanding achievements and commitment to playing a leading role in shaping the future of Australia. 